\title{
Moser functions and fractional Moser-Trudinger type inequalities
}

\author{
Ali Hyder* \\ Universität Basel \\ ali.hyder@unibas.ch
}

October 22, 2015

\begin{abstract}
We improve the sharpness of some fractional Moser-Trudinger type inequalities, particularly those studied by Lam-Lu and Martinazzi. As an application, improving upon works of Adimurthi and Lakkis, we prove the existence of weak solutions to the problem

$$
(-\Delta)^{\frac{n}{2}} u=\lambda u e^{b u^{2}} \text { in } \Omega, 0<\lambda<\lambda_{1}, b>0,
$$
\end{abstract}

with Dirichlet boundary condition, for any domain $\Omega$ in $\mathbb{R}^{n}$ with finite measure. Here $\lambda_{1}$ is the first eigenvalue of $(-\Delta)^{\frac{n}{2}}$ on $\Omega$.

\section{Introduction to the problem}

Let $n \geq 2$ and let $\Omega$ be a bounded domain in $\mathbb{R}^{n}$. The Sobolev embedding theorem states that $W_{0}^{k, p}(\Omega) \subset L^{q}(\Omega)$ for $1 \leq q \leq \frac{n p}{n-k p}$ and $k p<n$. However, it is not true that $W_{0}^{k, p}(\Omega) \subset L^{\infty}(\Omega)$ for $k p=n$. In the borderline case, as shown by Yudovich [28], Pohozaev [21] and Trudinger [27], $W_{0}^{1, n}(\Omega)$ embeds into an Orlich space and in fact

$$
\sup _{u \in W_{0}^{1, n}(\Omega),\|\nabla u\|_{L^{n}(\Omega)} \leq 1} \int_{\Omega} e^{\alpha|u|^{\frac{n}{n-1}}} d x<\infty,
$$

for some $\alpha>0$. Moser [20] found the best constant $\alpha$ in the inequality (1), obtaining the so called Moser-Trudinger inequality:

$$
\sup _{u \in W_{0}^{1, n}(\Omega),\|\nabla u\|_{L^{n}(\Omega)} \leq 1} \int_{\Omega} e^{\alpha_{n}|u|^{\frac{n}{n-1}}} d x<\infty, \quad \alpha_{n}=n\left|S^{n-1}\right|^{\frac{1}{n-1}} .
$$

${ }^{*}$ The author is supported by the Swiss National Science Foundation, project nr. PP00P2-144669. 
The constant $\alpha_{n}$ in (2) is the best constant in the sense that for any $\alpha>\alpha_{n}$, the supremum in (1) is infinite. A generalized version of Moser-Trudinger inequality is the following theorem of Adams [1]:

Theorem A ([1]) If $k$ is a positive integer less than $n$, then there is a constant $C=$ $C(k, n)$ such that

$$
\sup _{u \in C_{c}^{k}(\Omega),\left\|\nabla^{k} u\right\|_{L^{\frac{n}{k}(\Omega)}} \leq 1} \int_{\Omega} e^{\alpha|u|^{\frac{n}{n-k}}} d x \leq C|\Omega|,
$$

where

$$
\alpha=\alpha(k, n)=\frac{n}{\left|S^{n-1}\right|} \begin{cases}{\left[\frac{\pi^{\frac{n}{2}} 2^{k} \Gamma\left(\frac{k+1}{2}\right)}{\Gamma\left(\frac{n-k+1}{2}\right)}\right]^{\frac{n}{n-k}},} & m=\text { odd }, \\ {\left[\frac{\pi^{\frac{n}{2}} 2^{k} \Gamma\left(\frac{k}{2}\right)}{\Gamma\left(\frac{n-k}{2}\right)}\right]^{\frac{n}{n-k}},} & m=\text { even }\end{cases}
$$

and $\nabla^{k}:=\nabla \Delta^{\frac{k-1}{2}}$ for $k$ odd and $\nabla^{k}=\Delta^{\frac{k}{2}}$ for $k$ even. Moreover the constant $\alpha$ is sharp in the sense that

$$
\sup _{u \in C_{c}^{k}(\Omega),\left\|\nabla^{k} u\right\|_{L^{\frac{n}{k}(\Omega)}} \leq 1} \int_{\Omega} f(|u|) e^{\alpha|u|^{\frac{n}{n-k}}} d x=\infty,
$$

for any $f:[0, \infty) \rightarrow[0, \infty)$ with $\left.\lim _{t \rightarrow \infty} f(t)=\infty ! 1\right]$

In a recent work Martinazzi [17] has studied the Adams inequality in a fractional setting. In order to state its result first we recall the space

$$
L_{s}\left(\mathbb{R}^{n}\right):=\left\{u \in L_{l o c}^{1}\left(\mathbb{R}^{n}\right): \int_{\mathbb{R}^{n}} \frac{|u(x)|}{1+|x|^{n+2 s}} d x<\infty\right\} .
$$

The operator $(-\Delta)^{s}$ can be defined on the space $L_{s}\left(\mathbb{R}^{n}\right)$ via the duality

$$
\left\langle(-\Delta)^{s} u, \varphi\right\rangle:=\int_{\mathbb{R}^{n}} u(-\Delta)^{s} \varphi d x, \quad \varphi \in \mathcal{S}\left(\mathbb{R}^{n}\right),
$$

where

$$
(-\Delta)^{s} \varphi=\mathcal{F}^{-1}\left(|\xi|^{2 s} \hat{\varphi}\right), \quad \varphi \in \mathcal{S}\left(\mathbb{R}^{n}\right),
$$

$\mathcal{F}$ is the normalized Fourier transform and $\mathcal{S}\left(\mathbb{R}^{n}\right)$ is the Schwartz space. Notice that the integral in (4) is well-defined thanks to [7, Proposition 2.1].

Now for an open set $\Omega \subseteq \mathbb{R}^{n}$ (possibly $\Omega=\mathbb{R}^{n}$ ), $s>0$ and $1 \leq p \leq \infty$ we define the fractional Sobolev space $\tilde{H}^{s, p}(\Omega)$ by

$$
\tilde{H}^{s, p}(\Omega):=\left\{u \in L^{p}(\Omega): u=0 \text { on } \mathbb{R}^{n} \backslash \Omega,(-\Delta)^{\frac{s}{2}} u \in L^{p}\left(\mathbb{R}^{n}\right)\right\} .
$$

\footnotetext{
${ }^{1}$ Identity $(3)$ is proven in [1], although not explicitly stated.
} 
Theorem B $([\mathbf{1 7}])$ For any open set $\Omega \subset \mathbb{R}^{n}$ with finite measure and for any $p \in(1, \infty)$ we have

$$
\sup _{u \in \tilde{H}^{\frac{n}{p}, p}(\Omega),\left\|(-\Delta)^{\frac{n}{2 p}} u\right\|_{L^{p}(\Omega)} \leq 1} \int_{\Omega} e^{\alpha_{n, p}|u|^{p^{\prime}}} d x \leq C_{n, p}|\Omega|,
$$

where the constant $\alpha_{n, p}$ is given by

$$
\alpha_{n, p}=\frac{n}{\left|S^{n-1}\right|}\left(\frac{\Gamma\left(\frac{n}{2 p}\right) 2^{\frac{n}{p}} \pi^{\frac{n}{2}}}{\Gamma\left(\frac{n p-n}{2 p}\right)}\right)^{p^{\prime}}
$$

Moreover, the constant $\alpha_{n, p}$ is sharp in the sense that we cannot replace it with any larger one without making the above supremum infinite.

Notice that condition (3) in Theorem A is sharper than only requiring that the constant $\alpha$ in the exponential is sharp, as done in Theorem B. In fact Martinazzi asked whether it is true that

$$
\sup _{u \in \tilde{H}^{\frac{n}{p}, p}(\Omega),\left\|(-\Delta)^{\frac{n}{2 p}} u\right\|_{L^{p}(\Omega)} \leq 1} \int_{\Omega} f(|u|) e^{\alpha_{n, p}|u|^{p^{\prime}}} d x=\infty
$$

for any $f:[0, \infty) \rightarrow[0, \infty)$ with

$$
\lim _{t \rightarrow \infty} f(t)=\infty, \quad f \text { is Borel measurable, }
$$

and $\alpha_{n, p}$ is given by (5).

The point here is that Adams constructs smooth and compactly supported test functions similar to the standard Moser functions (constant in a small ball, and decaying logarithmically on an annulus), and then he estimates their $H_{0}^{k, \frac{n}{k}}$-norms in a very precise way. This becomes much more delicate when $k$ is not integer because instead of computing partial derivatives, one has to estimate the norms of fractional Laplacians (the term $\left\|(-\Delta)^{\frac{n}{2 p}} u\right\|_{L^{p}(\Omega)}$ in $\left.(6)\right)$. This is indeed done in [17], but the test functions introduced by Martinazzi are not efficient enough to prove (6). As we shall see this has consequences for applications to PDEs.

We shall prove that the answer to Martinazzi's question is positive, indeed in a slightly stronger form, namely the supremum in $(6)$ is infinite even if we consider the full $H^{\frac{n}{p}, p}$ norm on the whole space. More precisely we have:

Theorem 1.1 Let $\Omega$ be an open set in $\mathbb{R}^{n}$ with finite measure and let $f:[0, \infty) \rightarrow[0, \infty)$ satisfy (7). Then

$$
\sup _{u \in \tilde{H}^{\frac{n}{p}, p}(\Omega),\|u\|_{L^{p}(\Omega)}^{p}+\left\|(-\Delta)^{\frac{n}{2 p}} u\right\|_{L^{p}\left(\mathbb{R}^{n}\right)}^{p} \leq 1} \int_{\Omega} f(|u|) e^{\alpha_{n, p}|u|^{p^{\prime}}} d x=\infty, \quad 1<p<\infty,
$$

where the constant $\alpha_{n, p}$ is given by (5). 
The main difficulty in the proof of Theorem 1.1 is to construct test and cut-off functions in a way that their fractional Laplacians of suitable orders can be estimated precisely. This will be done in section 2 .

Here we mention that using a Green's representation formula, Iula-Maalaoui-Martinazzi 9] proved a particular case of Theorem 1.1 in one dimension. Their proof, though, does not extend to spaces $\tilde{H}^{\frac{n}{p}, p}(\Omega)$ when $\frac{n}{p}>1$ because the function constructed using the Green representation formula do not enjoy enough smoothness at the boundary. Trying to solve this with a smooth cut-off function at the boundary allows to prove (6) only when $f$ grows fast enough at infinity (for instance $f(t) \geq t^{a}$ for some $a>p^{\prime}$ ).

Now we move to Moser-Trudinger type inequalities on domains with infinite measure. In this direction we refer to [23, 11, 19] and the references there in. For our purpose, here we only state the work of Lam-Lu [11].

Theorem $\mathbf{C}([11])$ Let $p \in(1, \infty)$ and $\tau>0$. Then for every domain $\Omega \subset \mathbb{R}^{n}$ with finite measure, there exists $C=C(n, p, \tau)>0$ such that

$$
\sup _{u \in \tilde{H}^{\frac{n}{p}, p}\left(\mathbb{R}^{n}\right),\left\|(\tau I-\Delta)^{\frac{n}{2 p}} u\right\|_{L^{p}\left(\mathbb{R}^{n}\right)} \leq 1} \int_{\Omega} e^{\alpha_{n, p}|u|^{p^{\prime}}} d x \leq C|\Omega|,
$$

and

$$
\sup _{u \in \tilde{H}^{\frac{n}{p}, p}\left(\mathbb{R}^{n}\right),\left\|(\tau I-\Delta)^{\frac{n}{2 p}} u\right\|_{L^{p}\left(\mathbb{R}^{n}\right)} \leq 1} \int_{\mathbb{R}^{n}} \Phi\left(\alpha_{n, p}|u|^{p^{\prime}}\right) d x<\infty,
$$

where $\alpha_{n, p}$ is given by (5) and

$$
\Phi(t):=e^{t}-\sum_{j=0}^{j_{p}-2} \frac{t^{j}}{j !}, \quad j_{p}:=\min \{j \in \mathbb{N}: j \geq p\} .
$$

Furthermore, the constant $\alpha_{n, p}$ is sharp in the above inequalities, i.e., if $\alpha_{n, p}$ is replaced by any $\alpha>\alpha_{n, p}$, then the supremums are infinite.

In the spirit of Theorem 1.1 we prove a stronger version of the sharpness of the constant in Theorem $\mathrm{C}$.

Theorem 1.2 Let $\Omega \subset \mathbb{R}^{n}$ be a domain with finite measure and let $f:[0, \infty) \rightarrow[0, \infty)$ satisfy (7). Then for any $\tau>0$ and for any $p \in(1, \infty)$ we have (with the notations as in Theorem $C$ )

$$
\sup _{u \in \tilde{H}^{\frac{n}{p}, p}\left(\mathbb{R}^{n}\right),\left\|(\tau I-\Delta)^{\frac{n}{2 p}} u\right\|_{L^{p}\left(\mathbb{R}^{n}\right) \leq 1}} \int_{\Omega} f(|u|) e^{\alpha_{n, p}|u|^{p^{\prime}}} d x=\infty,
$$

and

$$
\sup _{u \in \tilde{H}^{\frac{n}{p}, p}\left(\mathbb{R}^{n}\right),\left\|(\tau I-\Delta)^{\frac{n}{2 p}} u\right\|_{L^{p}\left(\mathbb{R}^{n}\right)} \leq 1} \int_{\mathbb{R}^{n}} f(|u|) \Phi\left(\alpha_{n, p}|u|^{p^{\prime}}\right) d x=\infty
$$


As an application of Theorem 1.1 (in the case $p=2$ and $f(t)=t^{2}$, compare to (19) below) we prove the existence of (weak) solution to a semilinear elliptic equation with exponential nonlinearity. In order to state the theorem first we need the following definition.

Definition 1.1 Let $\Omega$ be an open set in $\mathbb{R}^{n}$ with finite measure. Let $f \in L^{p}(\Omega)$ for some $p \in(1, \infty)$. We say that $u$ is a weak solution of

$$
(-\Delta)^{\frac{n}{2}} u=f \text { in } \Omega
$$

if $u \in \tilde{H}^{\frac{n}{2}, 2}(\Omega)$ satisfies

$$
\int_{\mathbb{R}^{n}}(-\Delta)^{\frac{n}{4}} u(-\Delta)^{\frac{n}{4}} v d x=\int_{\Omega} f v d x \quad \text { for every } v \in \tilde{H}^{\frac{n}{2}, 2}(\Omega) .
$$

Theorem 1.3 Let $\Omega$ be an open set in $\mathbb{R}^{n}$ with finite measure. Let $0<\lambda<\lambda_{1}$ and $b>0$. Then there exists a nontrivial weak solution to the problem

$$
(-\Delta)^{\frac{n}{2}} u=\lambda u e^{b u^{2}} \text { in } \Omega
$$

Due to the fact that the embedding $\tilde{H}^{\frac{n}{2}, 2}(\Omega) \hookrightarrow L^{2}(\Omega)$ is compact for any open set $\Omega$ with finite measure (see Lemma A.7 in Appendix), we do not need any regularity assumption or boundedness assumption on the domain $\Omega$.

The equation (8) has been well studied by several authors in even and odd dimensions, with emphasis both on existence and compactness properties see e.g. [3, 5, 8, 10, 14, 15, 16, 18, 22, 26]. For instance, Lakkis [10], extending a work of Adimurthi [2], proved the existence of solution to (8) in any even dimension. In a recent work IannizzottoSquassina [8] have proven existence of nontrivial weak solution of (8) with $\Omega=(0,1)$ under an assumption, which turns out to be satisfied thanks to our Theorem 1.1, applied with $p=2$ (see Lemma 3.5).

\section{Moser type functions and proof of Theorems 1.1, 1.2}

We construct Moser type functions as follows:

First we fix two smooth functions $\eta$ and $\varphi$ such that $0 \leq \eta, \varphi \leq 1$,

$$
\eta \in C_{c}^{\infty}(-1,1), \quad \eta=1 \text { on }\left(-\frac{3}{4}, \frac{3}{4}\right)
$$

and

$$
\varphi \in C_{c}^{\infty}((-2,2)), \quad \varphi=1, \text { on }(-1,1)
$$


For $\varepsilon>0$, we set

$$
\psi_{\varepsilon}(t)= \begin{cases}1-\varphi_{\varepsilon}(t) & \text { if } 0 \leq t \leq \frac{1}{2} \\ \eta(t) & \text { if } t \geq \frac{1}{2}\end{cases}
$$

and

$$
v_{\varepsilon}(x)=\left(\log \frac{1}{\varepsilon}\right)^{-\frac{1}{p}}\left(\log \left(\frac{1}{\varepsilon}\right) \varphi_{\varepsilon}(|x|)+\log \left(\frac{1}{|x|}\right) \psi_{\varepsilon}(|x|)\right) \quad x \in \mathbb{R}^{n},
$$

where

$$
\varphi_{\varepsilon}(t)=\varphi\left(\frac{t}{\varepsilon}\right) .
$$

Our aim is to show that the supremums (in Theorems 1.1 and 1.2 taken over the functions $\left\{v_{\varepsilon}\right\}_{\varepsilon>0}$ (up to a proper normalization) are infinite.

The following proposition is crucial in the proof of Theorem 1.1

Proposition 2.1 Let

$$
u_{\varepsilon}(x):=\left|S^{n-1}\right|^{-\frac{1}{p}} 2^{\frac{n}{p^{\prime}}} \pi^{\frac{n}{2}} \Gamma\left(\frac{n}{2 p^{\prime}}\right) \frac{1}{\Gamma\left(\frac{n}{2 p}\right) \gamma_{n}} v_{\varepsilon}(x) .
$$

Then for $1<p<\infty$ there exists a constant $C>0$ such that

$$
\left\|(-\Delta)^{\frac{n}{2 p}} u_{\varepsilon}\right\|_{L^{p}\left(\mathbb{R}^{n}\right)} \leq\left(1+C\left(\log \frac{1}{\varepsilon}\right)^{-1}\right)^{\frac{1}{p}} .
$$

Proof. Since the proof of above proposition is quite trivial if $\frac{n}{2 p}$ is an integer, from now on we only consider the case when $\frac{n}{2 p}$ is not an integer.

From Lemmas 2.2 and 2.4 (below) we have

$$
\left\|(-\Delta)^{\frac{n}{2 p}} u_{\varepsilon}\right\|_{L^{p}\left(B_{3 \varepsilon} \cup B_{2}^{c}\right)}^{p} \leq C\left(\log \frac{1}{\varepsilon}\right)^{-1} .
$$

In order to estimate $(-\Delta)^{\sigma} v_{\varepsilon}$ on the domain $\{x: 3 \varepsilon<|x|<2\}$ we consider the function

$$
R_{\varepsilon}(x)=v_{\varepsilon}(x)-\left(\log \frac{1}{\varepsilon}\right)^{-\frac{1}{p}} \log \frac{1}{|x|}=: f_{\varepsilon}(x)+g_{\varepsilon}(x) \quad x \in \mathbb{R}^{n},
$$

where

$$
\begin{aligned}
f_{\varepsilon}(x): & = \begin{cases}v_{\varepsilon}(x)-\left(\log \frac{1}{\varepsilon}\right)^{-\frac{1}{p}} \log \frac{1}{|x|} & \text { if }|x|<2 \varepsilon \\
0 & \text { if }|x| \geq 2 \varepsilon\end{cases} \\
& =\left(\log \frac{1}{\varepsilon}\right)^{-\frac{1}{p}}\left(\log \frac{1}{\varepsilon}-\log \frac{1}{|x|}\right) \varphi_{\varepsilon}(|x|)
\end{aligned}
$$


and

$$
\begin{aligned}
g_{\varepsilon}(x): & = \begin{cases}v_{\varepsilon}(x)-\left(\log \frac{1}{\varepsilon}\right)^{-\frac{1}{p}} \log \frac{1}{|x|} & \text { if }|x|>\frac{1}{2} \\
0 & \text { if }|x| \leq \frac{1}{2}\end{cases} \\
& =\left(\log \frac{1}{\varepsilon}\right)^{-\frac{1}{p}}(\eta(|x|)-1) \log \frac{1}{|x|} .
\end{aligned}
$$

It is easy to see that for any $\sigma>0$

$$
\sup _{x \in \mathbb{R}^{n}}\left|(-\Delta)^{\sigma} g_{\varepsilon}(x)\right| \leq C\left(\log \frac{1}{\varepsilon}\right)^{-\frac{1}{p}} .
$$

With the help of Lemma A.8 and the triangle inequality we bound

$$
\begin{aligned}
\left|(-\Delta)^{\frac{n}{2 p}} u_{\varepsilon}(x)\right| & =\frac{1}{\left|S^{n-1}\right|^{\frac{1}{p}} \beta_{n, \frac{n}{2 p}}}\left|(-\Delta)^{\frac{n}{2 p}} R_{\varepsilon}(x)+\left(\log \frac{1}{\varepsilon}\right)^{-\frac{1}{p}}(-\Delta)^{\frac{n}{2 p}} \log \frac{1}{|x|}\right| \\
& \leq C\left|(-\Delta)^{\frac{n}{2 p}} R_{\varepsilon}(x)\right|+\left(\log \frac{1}{\varepsilon}\right)^{-\frac{1}{p}} \frac{1}{\left|S^{n-1}\right|^{\frac{1}{p}}} \frac{1}{|x|^{\frac{n}{p}}} .
\end{aligned}
$$

Using the elementary inequality

$$
(a+b)^{q} \leq a^{q}+C_{q}\left(b^{q}+a^{q-1} b\right), \quad 1 \leq q<\infty, a \geq 0, b \geq 0,
$$

we get

$$
\begin{aligned}
& \int_{3 \varepsilon<|x|<2}\left|(-\Delta)^{\frac{n}{2 p}} u_{\varepsilon}(x)\right|^{p} d x \\
& \leq \int_{3 \varepsilon<|x|<2}\left(\log \frac{1}{\varepsilon}\right)^{-1} \frac{1}{\left|S^{n-1}\right|} \frac{1}{|x|^{n}} d x+C \int_{3 \varepsilon<|x|<2}\left|(-\Delta)^{\frac{n}{2 p}} R_{\varepsilon}(x)\right|^{p} d x \\
& \quad+C\left(\log \frac{1}{\varepsilon}\right)^{-\frac{1}{p^{\prime}}} \int_{3 \varepsilon<|x|<2} \frac{1}{|x| \frac{n}{p^{\prime}}}\left|(-\Delta)^{\frac{n}{2 p}} R_{\varepsilon}(x)\right| d x \\
& \leq 1+C\left(\log \frac{1}{\varepsilon}\right)^{-1}+C\left(\log \frac{1}{\varepsilon}\right)^{-\frac{1}{p^{\prime}}} \int_{3 \varepsilon<|x|<2} \frac{1}{|x|^{\frac{n}{p^{\prime}}}}\left|(-\Delta)^{\frac{n}{2 p}} R_{\varepsilon}(x)\right| d x,
\end{aligned}
$$

where the last inequality follows from Lemma 2.3 (below). Using the pointwise estimate in Lemma 2.3 and (9) one can show that

$$
\int_{3 \varepsilon<|x|<2} \frac{1}{|x|^{\frac{n}{p^{\prime}}}}\left|(-\Delta)^{\frac{n}{2 p}} R_{\varepsilon}(x)\right| d x \leq C\left(\log \frac{1}{\varepsilon}\right)^{-\frac{1}{p}},
$$

which completes the proof. 
Lemma 2.2 Let $p \in(1, \infty)$. Then there exists a constant $C=C(n, p, \sigma)>0$ such that

$$
\left|(-\Delta)^{\sigma} v_{\varepsilon}(x)\right| \leq C\left(\log \frac{1}{\varepsilon}\right)^{-\frac{1}{p}} \varepsilon^{-2 \sigma} \quad \text { for }|x| \leq 3 \varepsilon, 0<\sigma<\frac{n}{2} .
$$

Moreover,

$$
\left\|(-\Delta)^{\frac{n}{2 p}} v_{\varepsilon}\right\|_{L^{p}\left(B_{3 \varepsilon}\right)}^{p} \leq C\left(\log \frac{1}{\varepsilon}\right)^{-1} .
$$

Proof. We claim that for every nonzero multiindex $\alpha \in \mathbb{N}^{n}$ there exists $C=C(n, \alpha)>0$ such that

$$
\left|D^{\alpha} v_{\varepsilon}(x)\right| \leq C\left(\log \frac{1}{\varepsilon}\right)^{-\frac{1}{p}} \varepsilon^{-|\alpha|}, \quad x \in \mathbb{R}^{n} .
$$

The claim follows from the fact that $D^{\alpha}\left(\varphi_{\varepsilon}+\psi_{\varepsilon}\right)=0$ on $B_{\frac{1}{2}}$ and hence we have the lemma if $\sigma$ is an integer. In the case when $\sigma$ is not a integer then we write $\sigma=m+s$ where $0<s<1$ and $m$ is an nonnegative integer. Then for $|x| \leq 3 \varepsilon$ we have (the following equivalent definition of fractional Laplacian can be found in [24, 4])

$$
\begin{gathered}
(-\Delta)^{\sigma} v_{\varepsilon}(x)=C_{n, s} \int_{\mathbb{R}^{n}} \frac{(-\Delta)^{m} v_{\varepsilon}(x+y)+(-\Delta)^{m} v_{\varepsilon}(x-y)-2(-\Delta)^{m} v_{\varepsilon}(x)}{|y|^{n+2 s}} d y . \\
A_{1}=\{x:|x| \leq 2 \varepsilon\}, \quad A_{2}=\left\{x: 2 \varepsilon<|x| \leq \frac{1}{4}\right\} \text { and } A_{3}=\left\{x:|x|>\frac{1}{4}\right\},
\end{gathered}
$$

we have

$$
(-\Delta)^{\sigma} v_{\varepsilon}(x)=C_{n, s} \sum_{i=1}^{3} I_{i}
$$

where

$$
I_{i}:=\int_{A_{i}} \frac{(-\Delta)^{m} v_{\varepsilon}(x+y)+(-\Delta)^{m} v_{\varepsilon}(x-y)-2(-\Delta)^{m} v_{\varepsilon}(x)}{|y|^{n+2 s}} d y .
$$

For $y \in A_{1}$, using 10$)$ we have

$$
\begin{aligned}
\left|(-\Delta)^{m} v_{\varepsilon}(x+y)+(-\Delta)^{m} v_{\varepsilon}(x-y)-2(-\Delta)^{m} v_{\varepsilon}(x)\right| & \leq|y|^{2}\left\|D^{2}(-\Delta)^{m} v_{\varepsilon}\right\|_{L^{\infty}} \\
& \leq C|y|^{2} \varepsilon^{-2 m-2}\left(\log \frac{1}{\varepsilon}\right)^{-\frac{1}{p}},
\end{aligned}
$$

and hence

$$
\left|I_{1}\right| \leq C \varepsilon^{-2 m-2}\left(\log \frac{1}{\varepsilon}\right)^{-\frac{1}{p}} \int_{A_{1}} \frac{d y}{|y|^{n+2 s-2}} \leq C\left(\log \frac{1}{\varepsilon}\right)^{-\frac{1}{p}} \varepsilon^{-2 \sigma} .
$$


For $m \geq 1$, again by 10

$$
\left|(-\Delta)^{m} v_{\varepsilon}(x+y)-(-\Delta)^{m} v_{\varepsilon}(x)\right| \leq C\left(\log \frac{1}{\varepsilon}\right)^{-\frac{1}{p}} \varepsilon^{-2 m} .
$$

Therefore,

$$
\left|I_{2}+I_{3}\right| \leq C\left(\log \frac{1}{\varepsilon}\right)^{-\frac{1}{p}} \varepsilon^{-2 m} \int_{|y|>\varepsilon} \frac{d y}{|y|^{n+2 s}} \leq C\left(\log \frac{1}{\varepsilon}\right)^{-\frac{1}{p}} \varepsilon^{-2 \sigma} .
$$

Since on $A_{2}|x+y| \leq 3 \varepsilon+\frac{1}{4}<\frac{1}{2}$, one has

$$
\begin{aligned}
& \left(\log \frac{1}{\varepsilon}\right)^{\frac{1}{p}}\left|v_{\varepsilon}(x+y)-v_{\varepsilon}(x)\right| \\
& =\mid \log \left(\frac{1}{\varepsilon}\right)\left(\varphi_{\varepsilon}(|x+y|)+\psi_{\varepsilon}(|x+y|)-\varphi_{\varepsilon}(|x|)-\psi_{\varepsilon}(|x|)\right) \\
& \quad+\log \left(\frac{\varepsilon}{|x+y|}\right) \psi_{\varepsilon}(|x+y|)-\log \left(\frac{\varepsilon}{|x|}\right) \psi_{\varepsilon}(|x|) \mid \\
& =\left|\log \left(\frac{\varepsilon}{|x+y|}\right) \psi_{\varepsilon}(|x+y|)-\log \left(\frac{\varepsilon}{|x|}\right) \psi_{\varepsilon}(|x|)\right| \\
& \leq C+\left|\log \left(\frac{\varepsilon}{|x+y|}\right) \psi_{\varepsilon}(|x+y|)\right|
\end{aligned}
$$

Hence, for $m=0$, changing the variable $y \mapsto \varepsilon z$

$$
\begin{aligned}
\left|I_{2}\right| & \leq C\left(\log \frac{1}{\varepsilon}\right)^{-\frac{1}{p}} \varepsilon^{-2 s}+C\left(\log \frac{1}{\varepsilon}\right)^{-\frac{1}{p}} \int_{\varepsilon<|y|<\frac{1}{4}} \frac{\left|\log \left(\frac{\varepsilon}{|x+y|}\right) \psi_{\varepsilon}(|x+y|)\right|}{|y|^{n+2 s}} d y \\
& \leq C\left(\log \frac{1}{\varepsilon}\right)^{-\frac{1}{p}} \varepsilon^{-2 s}+C\left(\log \frac{1}{\varepsilon}\right)^{-\frac{1}{p}} \varepsilon^{-2 s} \int_{|z|>1} \frac{|\log | \frac{x}{\varepsilon}+z|| \psi_{\varepsilon}\left(\varepsilon\left|\frac{x}{\varepsilon}+z\right|\right)}{|z|^{n+2 s}} d z \\
& \leq C\left(\log \frac{1}{\varepsilon}\right)^{-\frac{1}{p}} \varepsilon^{-2 s}+C\left(\log \frac{1}{\varepsilon}\right)^{-\frac{1}{p}} \varepsilon^{-2 s} \int_{|z|>1} \frac{\log (3+|z|)}{|z|^{n+2 s}} d z \\
& \leq C\left(\log \frac{1}{\varepsilon}\right)^{-\frac{1}{p}} \varepsilon^{-2 s} .
\end{aligned}
$$

Finally, for $m=0$, using that $\left|v_{\varepsilon}\right| \leq C\left(\log \frac{1}{\varepsilon}\right)^{-\frac{1}{p}}$ on $B_{\frac{1}{8}}^{c}$, we bound

$$
\left|I_{3}\right| \leq C\left(\log \frac{1}{\varepsilon}\right)^{-\frac{1}{p}} \int_{|y| \geq \frac{1}{4}} \frac{d y}{|y|^{n+2 s}} \leq C\left(\log \frac{1}{\varepsilon}\right)^{-\frac{1}{p}} .
$$

The lemma follows immediately. 
Lemma 2.3 For $|x| \geq 3 \varepsilon$ we have

$$
\left|(-\Delta)^{\sigma} f_{\varepsilon}(x)\right| \leq C \frac{1}{|x|^{2 \sigma}}\left(\log \frac{1}{\varepsilon}\right)^{-\frac{1}{p}} \begin{cases}\left(\frac{\varepsilon}{|x|}\right)^{n} & \text { if } 0<\sigma<1 \\ \left(\frac{\varepsilon}{|x|}\right)^{n-2 m} & \text { if } 1<\sigma=m+s<\frac{n}{2},\end{cases}
$$

where $m$ is a positive integer and $0<s<1$. In particular

$$
\left\|(-\Delta)^{\frac{n}{2 p}} R_{\varepsilon}\right\|_{L^{p}\left(B_{2} \backslash B_{3 \varepsilon}\right)} \leq C\left(\log \frac{1}{\varepsilon}\right)^{-\frac{1}{p}} .
$$

Proof. Notice that for every nonzero multiindex $\alpha \in \mathbb{N}^{n}$ we have

$$
\left|D^{\alpha} f_{\varepsilon}(x)\right| \leq C\left(\log \frac{1}{\varepsilon}\right)^{-\frac{1}{p}} \begin{cases}\frac{1}{\mid x x^{|\alpha|}} & \text { if }|x|<\varepsilon \\ \frac{1}{\varepsilon^{|\alpha|}} & \text { if } \varepsilon<|x| \leq 2 \varepsilon \\ 0 & \text { if }|x| \geq 2 \varepsilon\end{cases}
$$

First we consider $0<\sigma<1$. Using that $\left|\varphi_{\varepsilon}\right| \leq 1$, changing the variable $y \mapsto \varepsilon y$ and by Hölder inequality we obtain

$$
\begin{aligned}
\left|(-\Delta)^{\sigma} f_{\varepsilon}(x)\right| & =C\left|\int_{\mathbb{R}^{n}} \frac{f_{\varepsilon}(x)-f_{\varepsilon}(y)}{|x-y|^{n+2 \sigma}} d y\right| \\
& =C\left(\log \frac{1}{\varepsilon}\right)^{-\frac{1}{p}}\left|\int_{|y|<2 \varepsilon} \frac{\left(\log \frac{1}{\varepsilon}-\log \frac{1}{|y|}\right) \varphi_{\varepsilon}(|y|)}{|x-y|^{n+2 \sigma}} d y\right| \\
& \leq C \varepsilon^{n}\left(\log \frac{1}{\varepsilon}\right)^{-\frac{1}{p}}\left(\int_{|y|<2} \frac{d y}{|x-\varepsilon y|^{n p+2 p \sigma}}\right)^{\frac{1}{p}}\left(\left.\int_{|y|<2}|\log | y\right|^{p^{\prime}} d y\right)^{\frac{1}{p^{\prime}}} \\
& \leq C \varepsilon^{n}\left(\log \frac{1}{\varepsilon}\right)^{-\frac{1}{p}}\left(\frac{|x|^{n}}{\varepsilon^{n}} \frac{1}{|x|^{n p+2 p \sigma}} \int_{|y|<\frac{2 \varepsilon}{|x|}} \frac{d y}{\left|\frac{x}{|x|}-y\right|^{n p+2 p \sigma}}\right)^{\frac{1}{p}}, \\
& \leq C \frac{1}{|x|^{2 \sigma}}\left(\frac{\varepsilon}{|x|}\right)^{n}\left(\log \frac{1}{\varepsilon}\right)^{-\frac{1}{p}},
\end{aligned}
$$

where in the second last inequality we have used a change of variable $y \mapsto \frac{|x|}{\varepsilon} y$ and the last inequality follows from the uniform bound

$$
\frac{1}{\left|\frac{x}{|x|}-y\right|^{n p+2 p \sigma}} \leq C \text { for every }|x| \geq 3 \varepsilon,|y| \leq \frac{2 \varepsilon}{|x|} .
$$


For $\sigma>1$, changing the variable $y \mapsto|x| y$ and by (11) we have

$$
\begin{aligned}
\left|(-\Delta)^{\sigma} f_{\varepsilon}(x)\right| & =C\left|\int_{\mathbb{R}^{n}} \frac{(-\Delta)^{m} f_{\varepsilon}(x)-(-\Delta)^{m} f_{\varepsilon}(y)}{|x-y|^{n+2 s}} d y\right| \\
& =C\left|\int_{|y|<2 \varepsilon} \frac{(-\Delta)^{m} f_{\varepsilon}(y)}{|x-y|^{n+2 s}} d y\right| \\
& \leq C\left(\log \frac{1}{\varepsilon}\right)^{-\frac{1}{p}} \int_{|y|<2 \varepsilon} \frac{1}{|y|^{2 m}} \frac{1}{|x-y|^{n+2 s}} d y \\
& \leq C \frac{1}{|x|^{2 \sigma}}\left(\frac{\varepsilon}{|x|}\right)^{n-2 m}\left(\log \frac{1}{\varepsilon}\right)^{-\frac{1}{p}} .
\end{aligned}
$$

We conclude the lemma by $(9)$.

Lemma 2.4 For $0<\sigma<\frac{n}{2}$ there exists a constant $C=C(n, \sigma)$ such that

$$
\left|(-\Delta)^{\sigma} v_{\varepsilon}(x)\right| \leq C\left(\log \frac{1}{\varepsilon}\right)^{-\frac{1}{p}} \frac{1}{|x|^{n+2 \sigma}} \quad \text { for every } x \in B_{2}^{c} .
$$

Moreover,

$$
\left\|(-\Delta)^{\frac{n}{2 p}} v_{\varepsilon}\right\|_{L^{p}\left(B_{2}^{c}\right)}^{p} \leq C\left(\log \frac{1}{\varepsilon}\right)^{-1} .
$$

Proof. If $0<\sigma<1$ then

$$
\begin{aligned}
\left|(-\Delta)^{\sigma} v_{\varepsilon}(x)\right| & =C \int_{|y|<1} \frac{v_{\varepsilon}(y)}{|x-y|^{n+2 \sigma}} d y, \quad|x|>2 \\
& \leq C \frac{1}{|x|^{n+2 \sigma}} \int_{|y|<1} v_{\varepsilon}(y) d y \\
& \leq C\left(\log \frac{1}{\varepsilon}\right)^{-\frac{1}{p}} \frac{1}{|x|^{n+2 \sigma}} \int_{|y|<1}(\log |y|+\log 2) d y \\
& \leq C\left(\log \frac{1}{\varepsilon}\right)^{-\frac{1}{p}} \frac{1}{|x|^{n+2 \sigma}} .
\end{aligned}
$$

Since the integral in the right hand side of 12 is a proper integral, differentiating under the integral sign one can prove the lemma in a similar way.

Proof of Theorem 1.1 Without loss of generality we can assume that $B_{1} \subseteq \Omega$. Let $u_{\varepsilon}$ be defined as in Proposition 2.1. We claim that there exists a constant $\delta>0$ such that

$$
\limsup _{\varepsilon \rightarrow 0} \int_{B_{\varepsilon}} \exp \left(\frac{\alpha_{n, p}\left|u_{\varepsilon}\right|^{p^{\prime}}}{\left(\left\|u_{\varepsilon}\right\|_{L^{p}\left(\mathbb{R}^{n}\right)}^{p}+\left\|(-\Delta)^{\frac{n}{2 p}} u_{\varepsilon}\right\|_{L^{p}\left(\mathbb{R}^{n}\right)}^{p}\right)^{\frac{p^{\prime}}{p}}}\right) d x=: \limsup _{\varepsilon \rightarrow 0} I_{\varepsilon} \geq \delta .
$$


Then Theorem 1.1 follows at once, since $u_{\varepsilon} \rightarrow \infty$ on $B_{\varepsilon}$ as $\varepsilon \rightarrow 0$ and

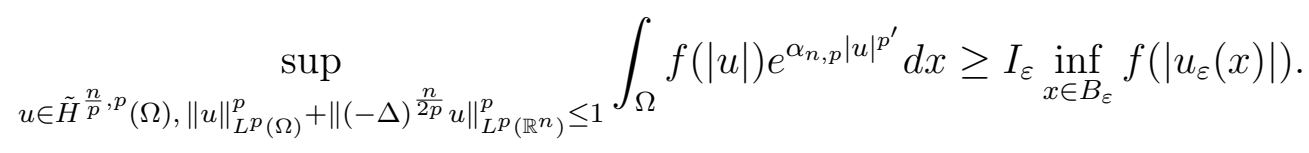

To prove (13) we choose $\varepsilon=e^{-k}$. Noticing that

$$
\begin{gathered}
\lim _{k \rightarrow \infty}-k+k\left(1+\frac{C}{k}\right)^{-\frac{p^{\prime}}{p}}=-C \frac{p^{\prime}}{p} \\
\left\|u_{\varepsilon}\right\|_{L^{p}\left(\mathbb{R}^{n}\right)}^{p} \leq C\left(\log \frac{1}{\varepsilon}\right)^{-1},
\end{gathered}
$$

and using Proposition 2.1 we have

$$
I_{\varepsilon} \geq\left|B_{1}\right| \varepsilon^{n} e^{n \log \frac{1}{\varepsilon}\left(1+C\left(\log \frac{1}{\varepsilon}\right)^{-1}\right)^{-\frac{p^{\prime}}{p}}}=\left|B_{1}\right| e^{-k n+k n\left(1+\frac{C}{k}\right)^{-\frac{p^{\prime}}{p}}} \geq \delta,
$$

for some $\delta>0$.

In order to prove Theorem 1.2 , first we prove the following proposition which gives a similar type of estimate as in Proposition 2.1.

Proposition 2.5 Let $\tau>0$ and $1<p<\infty$. Then there exists a constant $C>0$ such that

$$
\left\|(\tau I-\Delta)^{\frac{n}{2 p}} u_{\varepsilon}\right\|_{L^{p}\left(\mathbb{R}^{n}\right)} \leq\left(1+C\left(\log \frac{1}{\varepsilon}\right)^{-1}\right)^{\frac{1}{p}}
$$

Proof. We set

$$
w_{\varepsilon}(x)=(\tau I-\Delta)^{\frac{n}{2 p}} u_{\varepsilon}(x)-(-\Delta)^{\frac{n}{2 p}} u_{\varepsilon}(x) .
$$

We observe that there exists $C=C(p)>0$ such that

$$
h(t)=(1+t)^{p}-1-C\left(t^{p}+t^{p-1}+t^{\frac{1}{2}}\right)<0, \quad \text { for every } t>0,1 \leq p<\infty,
$$

which follows from the fact that $h(0)=0$ and $h^{\prime}(t)<0$ for every $t>0$. Therefore, there holds

$$
(a+b)^{p} \leq a^{p}+C_{p}\left(b^{p}+a b^{p-1}+b^{\frac{1}{2}} a^{p-\frac{1}{2}}\right), \quad a \geq 0, b \geq 0,1 \leq p<\infty,
$$


for some constant $C_{p}>0$ and using this inequality we bound

$$
\begin{aligned}
& \int_{\mathbb{R}^{n}}\left|(\tau I-\Delta)^{\frac{n}{2 p}} u_{\varepsilon}(x)\right|^{p} d x \\
& =\int_{\mathbb{R}^{n}}\left|w_{\varepsilon}(x)+(-\Delta)^{\frac{n}{2 p}} u_{\varepsilon}(x)\right|^{p} d x \\
& \leq \int_{\mathbb{R}^{n}}\left|(-\Delta)^{\frac{n}{2 p}} u_{\varepsilon}(x)\right|^{p}+C \int_{\mathbb{R}^{n}}\left|w_{\varepsilon}(x)\right|^{p} d x+C \int_{\mathbb{R}^{n}}\left|(-\Delta)^{\frac{n}{2 p}} u_{\varepsilon}(x)\right|\left|w_{\varepsilon}(x)\right|^{p-1} d x \\
& \quad+C \int_{\mathbb{R}^{n}}\left|(-\Delta)^{\frac{n}{2 p}} u_{\varepsilon}(x)\right|^{p-\frac{1}{2}}\left|w_{\varepsilon}(x)\right|^{\frac{1}{2}} d x \\
& =: I_{1}+I_{2}+I_{3}+I_{4} .
\end{aligned}
$$

From Proposition 2.1 we have

$$
I_{1} \leq 1+C\left(\log \frac{1}{\varepsilon}\right)^{-1}
$$

To estimate $I_{2}, I_{3}$ and $I_{4}$ we will use pointwise estimates on $(-\Delta)^{\sigma} u_{\varepsilon},(-\Delta)^{\sigma} w_{\varepsilon}$ and $L^{p}$ estimates on $(-\Delta)^{\sigma} w_{\varepsilon}$. For $0<\sigma<\frac{n}{2}$ combining Lemmas 2.2 - 2.4, A.8, and (9) we get

$$
\left|(-\Delta)^{\sigma} u_{\varepsilon}(x)\right| \leq C\left(\log \frac{1}{\varepsilon}\right)^{-\frac{1}{p}} \begin{cases}\varepsilon^{-2 \sigma} & \text { if }|x|<3 \varepsilon \\ |x|^{-2 \sigma} & \text { if } 3 \varepsilon<|x|<2 \\ |x|^{-n-2 \sigma} & \text { if }|x|>2\end{cases}
$$

With the help of (14) one can verify that

$$
\left\|(-\Delta)^{\sigma} u_{\varepsilon}\right\|_{L^{p}\left(\mathbb{R}^{n}\right)} \leq C(n, p, \sigma)\left(\log \frac{1}{\varepsilon}\right)^{-\frac{1}{p}}, \quad 1 \leq p<\infty, 0 \leq \sigma<\frac{n}{2 p},
$$

and together with Lemma A.2

$$
I_{2} \leq C\left(\log \frac{1}{\varepsilon}\right)^{-1}
$$

We conclude the proposition by showing that

$$
\int_{\mathbb{R}^{n}}\left|w_{\varepsilon}\right|^{q}\left|(-\Delta)^{\frac{n}{2 p}} v_{\varepsilon}\right|^{p-q} d x \leq C(n, p, q)\left(\log \frac{1}{\varepsilon}\right)^{-1}, \quad 0<q<\frac{p^{2}}{p+1} .
$$

It follows from Lemma A.1 that

$$
\left|w_{\varepsilon}(x)\right| \leq C\left(\log \frac{1}{\varepsilon}\right)^{-\frac{1}{p}}, \quad x \in \mathbb{R}^{n}, \frac{n}{2 p}<1,
$$


and for $\frac{n}{2 p}>1$

$$
\left|w_{\varepsilon}(x)\right| \leq C\left(\log \frac{1}{\varepsilon}\right)^{-\frac{1}{p}} \begin{cases}\varepsilon^{-\frac{n}{p}+2} & \text { if }|x|<3 \varepsilon \\ |x|^{-\frac{n}{p}+2} & \text { if } 3 \varepsilon<|x|<2 \\ 1 & \text { if }|x|>2,\end{cases}
$$

thanks to (14) and (15).

Splitting $\mathbb{R}^{n}$ into

$$
A_{1}=\{x:|x| \leq 2\} \quad \text { and } A_{3}=\{x:|x|>2\},
$$

we have

$$
\int_{\mathbb{R}^{n}}\left|w_{\varepsilon}\right|^{q}\left|(-\Delta)^{\frac{n}{2 p}} v_{\varepsilon}\right|^{p-q} d x=\sum_{i=1}^{2} J_{i}, \quad J_{i}:=\int_{A_{i}}\left|w_{\varepsilon}\right|^{q}\left|(-\Delta)^{\frac{n}{2 p}} v_{\varepsilon}\right|^{p-q} d x, i=1,2 .
$$

Using (14) one can show that $J_{1} \leq C\left(\log \frac{1}{\varepsilon}\right)^{-1}$ and together with $q<\frac{p^{2}}{p+1}$ one has $J_{3} \leq C\left(\log \frac{1}{\varepsilon}\right)^{-1}$, which gives 16 .

Proof of Theorem 1.2 Here also we can assume that $B_{1} \subseteq \Omega$. We choose $M>0$ large enough such that

$$
\Phi\left(\alpha_{n, p} t^{p^{\prime}}\right) \geq \frac{1}{2} e^{\alpha_{n, p} t^{p^{\prime}}}, \quad t \geq M .
$$

Then we have

$$
\begin{aligned}
& \int_{\mathbb{R}^{n}} f\left(\left|u_{\varepsilon}\right|\right) \Phi\left(\alpha_{n, p}\left|u_{\varepsilon}\right|^{p^{\prime}}\left\|(\tau I-\Delta)^{\frac{n}{2 p}} u_{\varepsilon}\right\|_{L^{p}\left(\mathbb{R}^{n}\right)}^{-p^{\prime}}\right) d x \\
& \geq \int_{u_{\varepsilon} \geq M} f\left(\left|u_{\varepsilon}\right|\right) \Phi\left(\alpha_{n, p}\left|u_{\varepsilon}\right|^{p^{\prime}}\left\|(\tau I-\Delta)^{\frac{n}{2 p}} u_{\varepsilon}\right\|_{L^{p}\left(\mathbb{R}^{n}\right)}^{-p^{\prime}}\right) d x \\
& \geq \frac{1}{2} \int_{B_{\varepsilon}} f\left(\left|u_{\varepsilon}\right|\right) e^{\alpha_{n, p}\left|u_{\varepsilon}\right| p^{\prime} \|(\tau I-\Delta)^{\frac{n}{2 p}}} u_{\varepsilon} \|_{L^{p}\left(\mathbb{R}^{n}\right)}^{p^{\prime}} d x,
\end{aligned}
$$

for $\varepsilon>0$ small enough. Now the proof follows as in Theorem 1.1, thanks to Proposition 2.5

\section{Proof of Theorem 1.3}

Throughout this section we use the notation $\|u\|=\left\|(-\Delta)^{\frac{n}{4}} u\right\|_{L^{2}\left(\mathbb{R}^{n}\right)}, H=\tilde{H}^{\frac{n}{2}, 2}(\Omega)$ and $\alpha_{0}=\alpha_{n, 2}$.

To prove Theorem 1.3 we follow the approach in [2, 10]. First we prove that $\lambda_{1}>0$, which makes the statement of Theorem 1.3 meaningful. 
Lemma 3.1 Let $\Omega$ be an open set in $\mathbb{R}^{n}$ with finite measure. Then $\lambda_{1}>0$ and there exists a function $u \in H$ such that

$$
\|u\|_{L^{2}(\Omega)}=1, \text { and }\|u\|^{2}=\lambda_{1} .
$$

Proof. We recall that

$$
\lambda_{1}=\inf \left\{\|u\|^{2}: u \in H,\|u\|_{L^{2}(\Omega)}=1\right\} .
$$

Let $\left\{u_{k}\right\}_{k=1}^{\infty} \subset H$ be a sequence such that

$$
\lim _{k \rightarrow \infty}\left\|u_{k}\right\|^{2}=\lambda_{1}, \quad\left\|u_{k}\right\|_{L^{2}(\Omega)}=1 \text { for every } k .
$$

Then up to a subsequence

$$
u_{k} \rightarrow u_{0} \text { in } H, \quad u_{k} \rightarrow u_{0} \text { in } L^{2}(\Omega),
$$

where the latter one follows from the compact embedding $H \hookrightarrow L^{2}(\Omega)$ (see Lemma A.7). Therefore,

$$
\lambda_{1} \leq\left\|u_{0}\right\|^{2} \leq \liminf _{k \rightarrow \infty}\left\|u_{k}\right\|^{2}=\lambda_{1}, \quad\left\|u_{0}\right\|_{L^{2}(\Omega)}=1 .
$$

Let us now define the functional

$$
J(u)=\frac{1}{2}\|u\|^{2}-\int_{\Omega} G(u) d x, \quad u \in H
$$

where

$$
G(t)=\int_{0}^{t} g(r) d r, \quad g(r):=\lambda r e^{b r^{2}}, \quad 0<\lambda<\lambda_{1}, b>0 .
$$

Then $J$ is $C^{2}$ and the Fréchet derivative of $J$ is be given by

$$
D J(u)(v)=\int_{\mathbb{R}^{n}}(-\Delta)^{\frac{n}{4}} u(-\Delta)^{\frac{n}{4}} v d x-\int_{\Omega} g(u) v d x, \quad v \in H .
$$

We also define

$$
\begin{gathered}
F(u)=D J(u)(u)=\|u\|^{2}-\int_{\Omega} g(u) u d x, \quad I(u)=J(u)-\frac{1}{2} F(u), \\
S=\{u \in H: u \neq 0, F(u)=0\} .
\end{gathered}
$$

Observe that if $u \in H$ is a nontrivial weak solution of (8) then $u \in S$.

With the above notations we have:

Lemma 3.2 The set $S$ is closed in the norm topology and

$$
0<s^{2}<\frac{\alpha_{0}}{b}, \quad s:=\sqrt{2 \inf _{u \in S} J(u)} .
$$


Proof. Since $F$ is continuous (actually $F$ is $C^{1}$ as $J$ is $C^{2}$ ), it is enough to show that 0 is an isolated point of $S$. If not, then there exists a sequence $\left\{u_{k}\right\} \subset S$ such that $\left\|u_{k}\right\| \rightarrow 0$ as $k \rightarrow \infty$. We set $v_{k}=\frac{u_{k}}{\left\|u_{k}\right\|}$. From the compactness of the embedding $H \hookrightarrow L^{q}(\Omega)$ for any $1 \leq q<\infty$, we can assume that (up to a subsequence) $v_{k} \rightarrow v$ in $H$ and $v_{k} \rightarrow v$ almost everywhere in $\Omega$. By Lemma 3.4 (below) we get

$$
1=\lambda \int_{\Omega} e^{b u_{k}^{2}} v_{k}^{2} d x \stackrel{k \rightarrow \infty}{\longrightarrow} \lambda \int_{\Omega} v^{2} d x \leq \lambda \frac{1}{\lambda_{1}}\|v\|^{2}<1
$$

which is a contradiction. Hence $S$ is closed.

Since,

$$
f(t):=\left(t^{2}-\frac{1}{b}\right) e^{b t^{2}}+\frac{1}{b}>0, \quad \text { for } t>0, b>0,
$$

which follows from $f(0)=0$ and $f^{\prime}(t)>0$ for $t>0$, we have

$$
I(u)=\frac{\lambda}{2} \int_{\Omega}\left(\left(u^{2}-\frac{1}{b}\right) e^{b u^{2}}+\frac{1}{b}\right) d x>0, \text { if } u \in H \backslash\{0\},
$$

and in particular $J(u)=I(u)>0$ for $u \in S$.

If possible, we assume that $s=0$. Then there exists a sequence $\left\{u_{k}\right\} \subset S$ such that $J\left(u_{k}\right) \rightarrow 0$ as $k \rightarrow \infty$. Moreover,

$$
\begin{aligned}
\left\|u_{k}\right\|^{2}=\lambda \int_{\Omega} u_{k}^{2} e^{b u_{k}^{2}} d x & =\lambda \int_{u_{k}^{2}>\frac{2}{b}} u_{k}^{2} e^{b u_{k}^{2}} d x+\lambda \int_{u_{k}^{2} \leq \frac{2}{b}} u_{k}^{2} e^{b u_{k}^{2}} d x \\
& \leq 4 \frac{\lambda}{2} \int_{u_{k}^{2}>\frac{2}{b}}\left(\left(u_{k}^{2}-\frac{1}{b}\right) e^{b u_{k}^{2}}+\frac{1}{b}\right) d x+\lambda \int_{u_{k}^{2} \leq \frac{2}{b}} u_{k}^{2} e^{b u_{k}^{2}} d x \\
& \leq 4 J\left(u_{k}\right)+\lambda \int_{u_{k}^{2} \leq \frac{2}{b}} u_{k}^{2} e^{b u_{k}^{2}} d x,
\end{aligned}
$$

and hence $u_{k}$ is bounded in $H$. Then up to a subsequence $u_{k} \rightarrow u$, a.e. in $\Omega$ and $u_{k} \rightarrow u$. Using Fatou lemma and ii) in Lemma 3.4 we obtain

$$
I(u)=\frac{\lambda}{2} \int_{\Omega}\left(\left(u^{2}-\frac{1}{b}\right) e^{b u^{2}}+\frac{1}{b}\right) d x \leq \liminf _{k \rightarrow \infty} I\left(u_{k}\right)=\liminf _{k \rightarrow \infty} J\left(u_{k}\right)=0,
$$

and hence $u=0$, thanks to (17). It follows from (18) that $u_{k} \rightarrow 0$ in $H$ which is a contradiction as $S$ is closed.

We prove now $s^{2}<\alpha_{0} b^{-1}$. First we fix $u \in H$ with $\|u\|=1$. We consider the function

$$
F_{u}(t):=F(t u)=\|t u\|^{2}-\lambda \int_{\Omega} t^{2} u^{2} e^{b t^{2} u^{2}} d x, \quad t \geq 0 .
$$

Then

$$
F_{u}(t) \geq t^{2}\left(\lambda_{1} \int_{\Omega} u^{2} d x-\lambda \int_{\Omega} u^{2} e^{b t^{2} u^{2}} d x\right)>0
$$


for $t>0$ sufficiently small and $\lim _{t \rightarrow \infty} F_{u}(t)=-\infty$. Hence, the continuity of $F_{u}$ implies that there exists $t_{u}>0$ such that $F_{u}\left(t_{u}\right)=0$, i.e., $t_{u} u \in S$. Thus

$$
\frac{s^{2}}{2} \leq J\left(t_{u} u\right) \leq \frac{1}{2}\left\|t_{u} u\right\|^{2}=\frac{1}{2} t_{u}^{2} .
$$

Again using that $t_{u} u \in S$ we have

$$
\int_{\Omega} u^{2} e^{b s^{2} u^{2}} d x \leq \frac{1}{\lambda t_{u}^{2}} \lambda \int_{\Omega}\left(t_{u} u\right)^{2} e^{b\left(t_{u} u\right)^{2}} d x=\frac{1}{\lambda t_{u}^{2}}\left\|t_{u} u\right\|^{2}=\frac{1}{\lambda}
$$

which implies that

$$
\sup _{\|u\| \leq 1, u \in H} \int_{\Omega} u^{2} e^{b s^{2} u^{2}} d x<\infty,
$$

and by Theorem 1.1 we deduce that $s^{2}<\alpha_{0} b^{-1}$.

Lemma 3.3 Let $u \in S$ be a minimizer of $J$ on $S$. Then $D J(u)=0$.

Proof. We fix a function $v \in H \backslash\{0\}$ and consider the function

$$
F_{u, v}(\gamma, t):=F(\gamma u+t v), \quad \gamma>0, t \in \mathbb{R}
$$

Differentiating $F_{u, v}$ with respect to $\gamma$ and using that $F(u)=0$, we get

$$
\frac{\partial F_{u, v}}{\partial \gamma}(1,0)=-2 b \lambda \int_{\mathbb{R}^{n}} u^{4} e^{b u^{2}} d x<0 .
$$

Hence, by implicit function theorem, there exists $\delta>0$ such that we can write $\gamma=\gamma(t)$ as a $C^{1}$ function of $t$ on the interval $(-\delta, \delta)$ which satisfies

$$
\gamma(0)=1, \quad F_{u, v}(\gamma(t), t)=0, \text { for every } t \in(-\delta, \delta) .
$$

Moreover, choosing $\delta>0$ smaller if necessary, we have $\gamma(t) u+t v \in S$ for every $t \in(-\delta, \delta)$. We write

$$
\begin{aligned}
D J(u)(v) & =\lim _{t \rightarrow 0} \frac{J(u+t v)-J(u)}{t} \\
& =\lim _{t \rightarrow 0}\left(\frac{J(\gamma(t) u+t v)-J(u)}{t}-\frac{J(\gamma(t) u+t v)-J(u+t v)}{t}\right) .
\end{aligned}
$$

Since $J$ is $C^{1}$, a first order expansion of $J$ yields

$$
\begin{aligned}
J(\gamma(t) u+t v)-J(u+t v) & =J((u+t v)+(\gamma(t)-1) u)-J(u+t v) \\
& =D J(u+t v)((\gamma(t)-1) u)+o((\gamma(t)-1)\|u\|) \\
& =(\gamma(t)-1) D J(u+t v)(u)+(\gamma(t)-1)\|u\| o(1) .
\end{aligned}
$$


Therefore, using that $F(u)=0$,

$$
\lim _{t \rightarrow 0} \frac{J(\gamma(t) u+t v)-J(u+t v)}{t}=\gamma^{\prime}(0) D J(u)(u)=0 .
$$

On the other hand, since $u$ is a minimizer of $J$ on $S$ and $\gamma(t) u+t v \in S$,

$$
\frac{J(\gamma(t) u+t v)-J(u)}{t}= \begin{cases}\geq 0 & \text { if } t \geq 0 \\ \leq 0 & \text { if } t \leq 0\end{cases}
$$

implies that (since it exists)

$$
\lim _{t \rightarrow 0} \frac{J(\gamma(t) u+t v)-J(u)}{t}=0
$$

This shows that $D J(u)(v)=0$ for every $v \in H$, i.e., $D J(u)=0$.

Proof of Theorem 1.3 Let $\left\{u_{k}\right\}$ be a sequence in $S$ such that $\lim _{k \rightarrow \infty} J\left(u_{k}\right) \rightarrow \frac{s^{2}}{2}$. Then by (18) $u_{k}$ is a bounded sequence in $H$ and consequently, up to a subsequence

$$
u_{k} \rightarrow u, \quad u_{k} \rightarrow u, \text { a.e. in } \Omega, \quad \ell:=\lim _{k \rightarrow \infty}\left\|u_{k}\right\|,
$$

for some $u \in H$. First we claim that $u \neq 0$.

Assuming $u=0$, by $i i$ ) in Lemma 3.4 (below) we get

$$
\lim _{k \rightarrow \infty}\left\|u_{k}\right\|^{2}=\lim _{k \rightarrow \infty} 2\left(J\left(u_{k}\right)+\frac{\lambda}{2 b} \int_{\Omega}\left(e^{b u_{k}^{2}}-1\right) d x\right)=s^{2}<\frac{\alpha_{0}}{b},
$$

and hence by $i$ ) in Lemma 3.4

$$
\lim _{k \rightarrow \infty}\left\|u_{k}\right\|^{2}=\lim _{k \rightarrow \infty} \lambda \int_{\Omega} u_{k}^{2} e^{b u_{k}^{2}} d x=0,
$$

a contradiction as $S$ is closed.

We claim that $\ell=\|u\|$. Then $u_{k} \rightarrow u$ in $H$ and applying Lemmas 3.2 and 3.3 we have Theorem 1.3 .

If the claim is false then necessarily we shall have $\ell>\|u\|$.

One has

$$
\begin{aligned}
\lim _{k \rightarrow \infty}\left\|u_{k}\right\|^{2} & =\lim _{k \rightarrow \infty} 2\left(J\left(u_{k}\right)+\frac{\lambda}{2 b} \int_{\Omega}\left(e^{b u_{k}^{2}}-1\right) d x\right) \\
& =2\left(\frac{s^{2}}{2}+\frac{\lambda}{2 b} \int_{\Omega}\left(e^{b u^{2}}-1\right) d x,\right) \\
& =s^{2}-2 J(u)+\|u\|^{2} .
\end{aligned}
$$

We divide the proof in two cases, namely $J(u) \leq 0$ and $J(u)>0$. 
Case 1. We consider that $J(u) \leq 0$. Since $u \neq 0$,

$$
\|u\|^{2} \leq \frac{\lambda}{b} \int_{\Omega}\left(e^{b u^{2}}-1\right) d x<\lambda \int_{\Omega} u^{2} e^{b u^{2}} d x
$$

where the second inequality follows from (17). It is easy to see that we can choose $0<t_{0}<1$ such that

$$
\left\|t_{0} u\right\|^{2}=\lambda \int_{\Omega}\left(t_{0} u\right)^{2} e^{b\left(t_{0} u\right)^{2}} d x
$$

that means $t_{0} u \in S$. Using that $I(t u)$ is strictly monotone increasing in $t$, which follows from the expression in (17), we obtain

$$
\frac{s^{2}}{2} \leq J\left(t_{0} u\right)=I\left(t_{0} u\right)<I(u) \leq \liminf _{k \rightarrow \infty} J\left(u_{k}\right)=\frac{s^{2}}{2}
$$

a contradiction.

Case 2. Here we assume that $J(u)>0$. Then

$$
\ell^{2}=\lim _{k \rightarrow \infty}\left\|u_{k}\right\|^{2}=s^{2}-2 J(u)+\|u\|^{2}<s^{2}+\|u\|^{2}<\frac{\alpha_{0}}{b}+\|u\|^{2} .
$$

Taking $v_{k}=\frac{u_{k}}{\left\|u_{k}\right\|}$ we see that (up to a subsequence)

$$
v_{k} \rightarrow v:=\frac{u}{\ell}, \quad v_{k} \rightarrow v, \text { a.e. in } \Omega,
$$

and by Lemma A.5, for every $p<\left(1-\|v\|^{2}\right)^{-1}$

$$
\sup _{k \in \mathbb{N}} \int_{\Omega} e^{p \alpha_{0} v_{k}^{2}} d x<\infty
$$

Taking (20) into account we have

$$
0<\ell^{2}-\|u\|^{2}=s^{2}-2 J(u)<\frac{\alpha_{0}}{b},
$$

and therefore, we can choose $\varepsilon_{0}>0$ such that

$$
1+\varepsilon_{0}=\frac{\alpha_{0}}{b} \frac{1}{\ell^{2}-\|u\|^{2}}, \quad \text { i.e., } \ell^{2}\left(1+\varepsilon_{0}\right)=\frac{\alpha_{0}}{b}\left(1-\frac{\|u\|^{2}}{\ell^{2}}\right)^{-1} .
$$

For $k$ large enough such that $\left\|u_{k}\right\|^{2} \leq \ell^{2}\left(1+\frac{\varepsilon_{0}}{2}\right)$ holds, we observe that $b\left\|u_{k}\right\|^{2} \leq p_{0} \alpha_{0}$ for some $1<p_{0}<\left(1-\|v\|^{2}\right)^{-1}$. Thus, for some $p_{1}>1, p_{2}>1$ with $p_{1} p_{2} p_{0}<\left(1-\|v\|^{2}\right)^{-1}$ we obtain

$$
\sup _{k \in \mathbb{N}} \int_{\Omega}\left(u_{k}^{2} e^{b u_{k}^{2}}\right)^{p_{1}} d x \leq \sup _{k \in \mathbb{N}}\left\|u_{k}^{2 p_{1}}\right\|_{L^{p_{2}^{\prime}(\Omega)}}\left\|e^{p_{1} p_{0} \alpha_{0} v_{k}^{2}}\right\|_{L^{p_{2}(\Omega)}}<\infty
$$


and together with Lemma A.9

$$
\lim _{k \rightarrow \infty} \int_{\Omega} u_{k}^{2} e^{b u_{k}^{2}} d x=\int_{\Omega} u^{2} e^{b u^{2}} d x
$$

Indeed,

$$
\|u\|^{2}<\ell^{2}=\lim _{k \rightarrow \infty}\left\|u_{k}\right\|^{2}=\lambda \lim _{k \rightarrow \infty} \int_{\Omega} u_{k}^{2} e^{b u_{k}^{2}} d x=\lambda \int_{\Omega} u^{2} e^{b u^{2}} d x
$$

and we can now proceed as in Case 1.

Lemma 3.4 Let $u_{k}, v_{k} \in H$ such that $u_{k} \rightarrow u$ in $H, u_{k} \rightarrow u$, a.e. in $\Omega, v_{k} \rightarrow v$ in $H$ and $v_{k} \rightarrow v$, a.e. in $\Omega$. Then

i) If

$$
\limsup _{k \rightarrow \infty}\left\|u_{k}\right\|^{2}<\frac{\alpha_{0}}{b}
$$

then for every integer $\ell \geq 1$

$$
\lim _{k \rightarrow \infty} \int_{\Omega} e^{b u_{k}^{2}} v_{k}^{\ell} d x=\int_{\Omega} e^{b u^{2}} v^{\ell} d x
$$

ii) If

$$
\limsup _{k \rightarrow \infty} \int_{\Omega} u_{k}^{2} e^{b u_{k}^{2}} d x<\infty
$$

then

$$
\lim _{k \rightarrow \infty} \int_{\Omega} e^{b u_{k}^{2}} d x=\int_{\Omega} e^{b u^{2}} d x
$$

Proof. We prove the lemma with the help of Lemma A.9 (in Appendix).

We choose $p>1$ such that for $k$ large enough $p\left\|u_{k}\right\|^{2}<\frac{\alpha_{0}}{b}$ holds and together with Theorem $\mathrm{C}$ we have

$$
\sup _{k \in \mathbb{N}} \int_{\Omega} e^{p b u_{k}^{2}} d x<\infty
$$

Since the embedding $\tilde{H}^{\frac{n}{2}, 2}(\Omega) \hookrightarrow L^{q}(\Omega)$ is compact (see Lemma A.7) for every $1 \leq q<\infty$, we have

$$
v_{k}^{q} \rightarrow v^{q} \text { in } L^{1}(\Omega) .
$$

Indeed,

$$
\sup _{k \in \mathbb{N}}\left\|e^{b u_{k}^{2}} v_{k}^{\ell}\right\|_{L^{p}(\Omega)} \leq\left\|v_{k}^{\ell}\right\|_{L^{p^{\prime}}(\Omega)}\left\|e^{b u_{k}^{2}}\right\|_{L^{p}(\Omega)}<\infty,
$$

and we conclude $i$ ).

Now $i i$ ) follows from

$$
\int_{u_{k}^{2}>M} e^{b u_{k}^{2}} d x \leq \frac{1}{M} \int_{u_{k}^{2}>M} u_{k}^{2} e^{b u_{k}^{2}} d x \leq \frac{C}{M},
$$


which implies that the function $f_{k}:=e^{b u_{k}^{2}}$ satisfies the condition $\left.i i\right)$ in Lemma A.9.

In the following lemma we prove that the assumption $H^{\prime}(v)$ in [8] is true under certain conditions.

Lemma 3.5 Let $\alpha_{0}>0$. Let $f(t)=e^{\alpha_{0} t^{2}} h(t)$ satisfies $H(i)-($ iii $)$ in [8]. Let $h \geq 0$ on $[0, \infty)$ and $h(-t)=-h(t)$. Let $s \frac{f(s t)}{t}$ be a monotone increasing function with respect to $t$ on $(0, \infty), s \neq 0$. If $\lim _{t \rightarrow \infty} h(t) t=\infty$ then there exists $u \in \tilde{H}^{\frac{1}{2}, 2}((0,1))$ such that $\sqrt{2 \pi}\left\|(-\Delta)^{\frac{1}{4}} u\right\|_{L^{2}(\mathbb{R})}=1$ and

$$
\sup _{t>0} \Phi(t u):=\sup _{t>0}\left(\frac{t^{2}}{4 \pi}-\int_{0}^{1} F(t u) d x\right)<\frac{\omega}{2 \alpha_{0}},
$$

where

$$
F(t)=\int_{0}^{t} f(s) d s
$$

and $\omega$ is as in [8].

Proof. For a given $M>0$ we can choose $u \in \tilde{H}^{\frac{1}{2}, 2}((0,1))$ such that

$$
\int_{0}^{1} f\left(\sqrt{\frac{2 \pi^{2}}{\alpha_{0}}} u\right) u d x>M, \quad \sqrt{2 \pi}\left\|(-\Delta)^{\frac{1}{4}} u\right\|_{L^{2}(\mathbb{R})}=1,
$$

thanks to Theorem 1.1. Differentiating with respect to $t$ one has

$$
\Phi^{\prime}(t u)=t\left(\frac{1}{2 \pi}-\int_{0}^{1} \frac{f(t u)}{t} u d x\right) .
$$

Hence, for $t \geq \sqrt{\frac{2 \pi^{2}}{\alpha_{0}}}=: t_{0}$ and $2 \pi M>t_{0}$

$$
\Phi^{\prime}(t u) \leq t\left(\frac{1}{2 \pi}-\int_{0}^{1} \frac{f\left(t_{0} u\right)}{t_{0}} u d x\right)<0 .
$$

Thus $\Phi^{\prime}(t u) \leq 0$ on $\left(t_{0}-\varepsilon, \infty\right)$ for some $\varepsilon>0$ and therefore,

$$
\sup _{t>0} \Phi(t u)=\sup _{t \in\left(0, t_{0}-\varepsilon\right)} \Phi(t u) \leq \sup _{t \in\left(0, t_{0}-\varepsilon\right)} \frac{t^{2}}{4 \pi}<\frac{\pi}{2 \alpha_{0}} .
$$

Since $\omega=\pi$, thanks to Theorem B, we conclude the lemma. 


\section{A Appendix}

Lemma A.1 (Pointwise estimate) Let $s>0$ and not an integer. Let $m$ be the smallest integer greater than $s$. Then for any $\tau>0$

$$
\left|(\tau I-\Delta)^{s} u(x)-(-\Delta)^{s} u(x)\right| \leq C \sum_{j=1}^{m-1}\left|(-\Delta)^{s-j} u(x)\right|+C\left\|(-\Delta)^{\sigma} u\right\|_{L^{1}\left(\mathbb{R}^{n}\right)}, \quad u \in \mathcal{S}\left(\mathbb{R}^{n}\right),
$$

where $\sigma \in\left(\max \left\{\frac{n}{2}-m+s, 0\right\}, \frac{n}{2}\right)$, the constant $C$ depends only on $n, s, \sigma, \tau$ and for $m=1$ the above sum can be interpreted as zero.

Proof. We set $f(t)=t^{s}$ on $\mathbb{R}^{+}$. By Taylor's expansion we have

$f(t+\tau)=f(t)+\tau f^{\prime}(t)+\cdots+\frac{\tau^{m-1}}{(m-1) !} f^{m-1}(t)+\frac{\tau^{m}}{m !} f^{m}\left(\xi_{t}\right), \quad$ for some $t<\xi_{t}<t+\tau$.

In particular

$$
\left(\tau+t^{2}\right)^{s}=t^{2 s}+c_{1} t^{2 s-2}+c_{2} t^{2 s-4}+\cdots+c_{m-1} t^{2 s-2 m+2}+E(t),
$$

where the function $E$ satisfies the estimate

$$
|E(t)| \leq C(1+t)^{2 s-2 m}, \quad t>0 .
$$

Therefore, for $u \in \mathcal{S}\left(\mathbb{R}^{n}\right)$

$$
\begin{aligned}
\mathcal{F}\left((\tau I-\Delta)^{s} u\right)(\xi) & =\left(\tau+|\xi|^{2}\right)^{s} \hat{u} \\
& =\left(|\xi|^{2 s}+c_{1}|\xi|^{2 s-2}+\cdots+c_{m-1}|\xi|^{2 s-2 m+2}+E(|\xi|)\right) \hat{u} \\
& =\sum_{j=0}^{m-1} c_{j}|\xi|^{2 s-2 j} \hat{u}+E(|\xi|) \hat{u}(\xi) \\
& =\sum_{j=0}^{m-1} c_{j} \mathcal{F}\left((-\Delta)^{s-j} u\right)+E(|\xi|) \hat{u}(\xi),
\end{aligned}
$$

and hence

$$
(\tau I-\Delta)^{s} u(x)=\sum_{j=0}^{m-1} c_{j}(-\Delta)^{s-j} u(x)+\mathcal{F}^{-1}(E \hat{u})(x) .
$$

To estimate the term $\mathcal{F}^{-1}(E \hat{u})$ (uniformly in $x$ ) in terms of $L^{1}\left(\mathbb{R}^{n}\right)$ norm of (fractional) derivative of $u$, we observe that

$$
\begin{aligned}
|E(|\xi|) \hat{u}(\xi)| & =\left|E(|\xi|) \frac{1}{|\xi|^{2 \sigma}} \widehat{(-\Delta)^{\sigma}} u(\xi)\right| \\
& \leq \frac{C}{|\xi|^{2 \sigma}\left(1+|\xi|^{2}\right)^{m-s}}\left|\widehat{(-\Delta)^{\sigma}} u(\xi)\right| \\
& \leq \frac{C}{|\xi|^{2 \sigma}\left(1+|\xi|^{2}\right)^{m-s}}\left\|(-\Delta)^{\sigma} u\right\|_{L^{1}\left(\mathbb{R}^{n}\right)} .
\end{aligned}
$$


Thus

$$
\left|\mathcal{F}^{-1}(E \hat{u})(x)\right| \leq C\|E \hat{u}\|_{L^{1}\left(\mathbb{R}^{n}\right)} \leq C\left\|(-\Delta)^{\sigma} u\right\|_{L^{1}\left(\mathbb{R}^{n}\right)},
$$

and we complete the proof.

Lemma A.2 ( $L^{p}$ Estimate) Let $s>0$ be a noninteger. Let $\tau>0$ be any fixed number. Then for $p \in(1, \infty)$ there exists $C=C(n, s, p, \tau)>0$ such that

$$
\left\|(\tau I-\Delta)^{s} u-(-\Delta)^{s} u\right\|_{L^{p}\left(\mathbb{R}^{n}\right)} \leq C \begin{cases}\|u\|_{L^{p}\left(\mathbb{R}^{n}\right)} & \text { if } s<1 \\ \left\|u+(-\Delta)^{s-1} u\right\|_{L^{p}\left(\mathbb{R}^{n}\right)} & \text { if } s>1\end{cases}
$$

Proof. We have

$$
\begin{aligned}
\mathcal{F}\left((\tau I-\Delta)^{s} u\right)(\xi)-\mathcal{F}\left((-\Delta)^{s} u\right)(\xi) & =\left(\left(\tau+|\xi|^{2}\right)^{s}-|\xi|^{2 s}\right) \hat{u}(\xi) \\
& = \begin{cases}\left(\left(\tau+|\xi|^{2}\right)^{s}-|\xi|^{2 s}\right) \hat{u}(\xi) & \text { if } s<1 \\
\frac{\left(\tau+|\xi|^{2}\right)^{s}-|\xi|^{2 s}}{1+|\xi|^{2 s-2}}\left(1+|\xi|^{2 s-2}\right) \hat{u}(\xi) & \text { if } s>1\end{cases} \\
& =: \begin{cases}m(\xi) \hat{u}(\xi) & \text { if } s<1 \\
m(\xi) \mathcal{F}\left(u+(-\Delta)^{s-1} u\right)(\xi) & \text { if } s>1 .\end{cases}
\end{aligned}
$$

Now the proof follows from the Hormander multiplier theorem (see [25, p. 96]).

The following lemma appears already in [6, p. 46], but for the reader's convenience we give a more detailed proof.

Lemma A.3 (Equivalence of norms) Let $\sigma>0$. Then for $p \in(1, \infty)$ there exists a constant $C>0$ such that for every $u \in \mathcal{S}\left(\mathbb{R}^{n}\right)$

$$
\begin{aligned}
\frac{1}{C}\left(\|u\|_{L^{p}\left(\mathbb{R}^{n}\right)}+\left\|(-\Delta)^{\sigma} u\right\|_{L^{p}\left(\mathbb{R}^{n}\right)}\right) & \leq\left\|(I-\Delta)^{\sigma} u\right\|_{L^{p}\left(\mathbb{R}^{n}\right)} \\
& \leq C\left(\|u\|_{L^{p}\left(\mathbb{R}^{n}\right)}+\left\|(-\Delta)^{\sigma} u\right\|_{L^{p}\left(\mathbb{R}^{n}\right)}\right) .
\end{aligned}
$$

Proof. We set

$$
G_{\sigma}(x)=\frac{1}{(4 \pi)^{\frac{\sigma}{2}}} \frac{1}{\Gamma\left(\frac{\sigma}{2}\right)} \int_{0}^{\infty} e^{-\pi \frac{|x|^{2}}{t}} e^{-\frac{t}{4 \pi}} t^{\frac{-n+\sigma}{2}} \frac{d t}{t}
$$

which is the Bessel potential of order $\sigma$ (see [25, p. 130]). Then

$$
\int_{\mathbb{R}^{n}} G_{\sigma}(x) d x=1, \quad \hat{G}_{\sigma}(x)=\frac{1}{(2 \pi)^{\frac{n}{2}}} \frac{1}{\left(1+|x|^{2}\right)^{\frac{\sigma}{2}}} .
$$

Setting $f=(I-\Delta)^{\sigma} u$ we can write $u=G_{2 \sigma} * f$ and by Young's inequality one has $\|u\|_{L^{p}\left(\mathbb{R}^{n}\right)} \leq\|f\|_{L^{p}\left(\mathbb{R}^{n}\right)}$. Again writing $u=G_{2 \sigma} * f$ and taking Fourier transform we obtain

$$
\mathcal{F}\left((-\Delta)^{\sigma} u\right)=|\xi|^{2 \sigma} \hat{u}=|\xi|^{2 \sigma} \frac{1}{\left(1+|\xi|^{2}\right)^{\sigma}} \hat{f}=: m(\xi) \hat{f}
$$


and by Hormander multiplier theorem we get $\left\|(-\Delta)^{\sigma} u\right\|_{L^{p}\left(\mathbb{R}^{n}\right)} \leq C\|f\|_{L^{p}\left(\mathbb{R}^{n}\right)}$. Thus,

$$
\|u\|_{L^{p}\left(\mathbb{R}^{n}\right)}+\left\|(-\Delta)^{\sigma} u\right\|_{L^{p}\left(\mathbb{R}^{n}\right)} \leq C\left\|(I-\Delta)^{\sigma} u\right\|_{L^{p}\left(\mathbb{R}^{n}\right)} .
$$

To conclude the lemma, it is sufficient to show that

$$
\left\|(-\Delta)^{s} u\right\|_{L^{p}\left(\mathbb{R}^{n}\right)} \leq C(n, s, \sigma, p)\left(\|u\|_{L^{p}\left(\mathbb{R}^{n}\right)}+\left\|(-\Delta)^{\sigma} u\right\|_{L^{p}\left(\mathbb{R}^{n}\right)}\right), \quad 0<s<\sigma,
$$

thanks to Lemma A.2.

In order to prove (21) we fix a function $\varphi \in C_{c}^{\infty}\left(B_{2}\right)$ such that $\varphi=1$ on $B_{1}$. Then

$$
\mathcal{F}\left((-\Delta)^{s} u\right)=|\xi|^{2 s} \hat{u}=|\xi|^{2 s} \varphi \hat{u}+|\xi|^{2 s}(1-\varphi) \hat{u}=m_{1}(\xi) \hat{u}+m_{2}(\xi) \mathcal{F}\left((-\Delta)^{\sigma} u\right),
$$

where $m_{1}(\xi)=|\xi|^{2 s} \varphi(\xi), m_{2}(\xi)=|\xi|^{2 s-2 \sigma}(1-\varphi(\xi))$ are multipliers and we conclude (21) by Hormander multiplier theorem.

Lemma A.4 (Embedding to an Orlicz space) Let $\Omega$ be an open set with finite measure. Then for every $u \in \tilde{H}^{\frac{n}{2}, 2}(\Omega)$

$$
\int_{\Omega} e^{u^{2}} d x<\infty
$$

Proof. We set $f=(-\Delta)^{\frac{n}{4}} u$. By [17, Proposition 8] we have

$$
u(x)=\int_{\Omega} G(x, y) f(y) d y, \quad 0 \leq G(x, y) \leq \frac{C_{n}}{|x-y|^{\frac{n}{2}}},
$$

where $G$ is a Greens function.

We choose $M>0$ large enough such that $\|\tilde{f}\|_{L^{2}} C_{n}<\alpha_{0}$, where $\tilde{f}=f-f \chi_{\{|f| \leq M\}}$. Then

$$
|u(x)| \leq C(M)+C_{n} I_{\frac{n}{2}} \tilde{f}(x), \quad I_{\frac{n}{2}} \tilde{f}(x):=\int_{\Omega} \frac{|\tilde{f}(y)|}{|x-y|^{\frac{n}{2}}} d y,
$$

and by [1, Theorem 2] we conclude the proof.

As a consequence of the above lemma one can prove a higher dimensional generalization of Lions lemma [13] (for a simple proof see e.g. [8, Lemma 2.6]), namely

Lemma A.5 (Lions) Let $u_{k}$ be a sequence in $\tilde{H}^{\frac{n}{2}, 2}(\Omega)$ such that

$$
u_{k} \rightarrow u \text { in } \tilde{H}^{\frac{n}{2}, 2}(\Omega), \quad 0<\left\|(-\Delta)^{\frac{n}{4}} u\right\|_{L^{2}\left(\mathbb{R}^{n}\right)}<1, \quad\left\|(-\Delta)^{\frac{n}{4}} u_{k}\right\|_{L^{2}\left(\mathbb{R}^{n}\right)}=1 .
$$

Then for every $0<p<\left(1-\left\|(-\Delta)^{\frac{n}{4}} u\right\|_{L^{2}\left(\mathbb{R}^{n}\right)}^{2}\right)^{-1}$, the sequence $\left\{e^{\alpha_{0} p u_{k}}\right\}_{1}^{\infty}$ is bounded in $L^{1}(\Omega)$. 
Lemma A.6 (Poincaré inequality) Let $\Omega$ be an open set with finite measure. Then there exists a constant $C>0$ such that

$$
\|u\|_{L^{2}(\Omega)} \leq C\left\|(-\Delta)^{\frac{s}{2}} u\right\|_{L^{2}\left(\mathbb{R}^{n}\right)}, \text { for every } u \in \tilde{H}^{s, 2}(\Omega)
$$

Proof. We have

$$
|\hat{u}(\xi)| \leq \frac{1}{(2 \pi)^{\frac{n}{2}}}\|u\|_{L^{1}(\Omega)} \leq \frac{1}{(2 \pi)^{\frac{n}{2}}}|\Omega|^{\frac{1}{2}}\|u\|_{L^{2}(\Omega)},
$$

and hence

$$
\begin{aligned}
\|u\|_{L^{2}(\Omega)}^{2} & =\int_{\mathbb{R}^{n}}|\hat{u}|^{2} d \xi=\int_{|\xi|<\delta}|\hat{u}|^{2} d \xi+\int_{|\xi| \geq \delta}|\hat{u}|^{2} d \xi \\
& \leq \frac{1}{(2 \pi)^{n}}|\Omega|\|u\|_{L^{2}(\Omega)}^{2}\left|B_{1}\right| \delta^{n}+\delta^{-2 s} \int_{|\xi| \geq \delta}|\xi|^{2 s}|\hat{u}|^{2} d \xi \\
& \leq \frac{1}{(2 \pi)^{n}}|\Omega|\left|B_{1}\right| \delta^{n}\|u\|_{L^{2}(\Omega)}^{2}+\delta^{-2 s} \int_{\mathbb{R}^{n}}\left|\mathcal{F}\left((-\Delta)^{\frac{s}{2}} u\right)(\xi)\right|^{2} d \xi
\end{aligned}
$$

Choosing $\delta>0$ so that $\frac{1}{(2 \pi)^{n}}|\Omega|\left|B_{1}\right| \delta^{n}=\frac{1}{2}$ we complete the proof.

Lemma A.7 (Compact embedding) Let $\Omega$ be an open set in $\mathbb{R}^{n}$ with finite measure. Then the embedding $\tilde{H}^{s, 2}(\Omega) \hookrightarrow \tilde{H}^{r, 2}(\Omega)$ is compact for any $0 \leq r<s$ (with the notation $\left.\tilde{H}^{0,2}(\Omega)=L^{2}(\Omega)\right)$. Moreover, $\tilde{H}^{\frac{n}{2}, 2}(\Omega) \hookrightarrow L^{p}(\Omega)$ is compact for any $p \in[1, \infty)$.

Proof. We prove the lemma in few steps.

Step 1 The embedding $\tilde{H}^{s, 2}(\Omega) \hookrightarrow \tilde{H}^{r, 2}(\Omega)$ is continuous for any $0 \leq r<s$.

With the notation $(-\Delta)^{0} u=u$ we see that

$$
\begin{aligned}
\left\|(-\Delta)^{\frac{r}{2}} u\right\|_{L^{2}\left(\mathbb{R}^{n}\right)}^{2} & =\int_{\mathbb{R}^{n}}|\xi|^{2 r}|\hat{u}|^{2} d \xi=\int_{|\xi| \leq 1}|\xi|^{2 r}|\hat{u}|^{2} d \xi+\int_{|\xi|>1}|\xi|^{2 r}|\hat{u}|^{2} d \xi \\
& \leq \int_{|\xi| \leq 1}|\hat{u}|^{2} d \xi+\int_{|\xi|>1}|\xi|^{2 s}|\hat{u}|^{2} d \xi \leq\|u\|_{L^{2}(\Omega)}^{2}+\left\|(-\Delta)^{\frac{s}{2}} u\right\|_{L^{2}\left(\mathbb{R}^{n}\right)}^{2},
\end{aligned}
$$

which is Step 1, thanks to Lemma A.6

Step 2 For a given $s>0$ and a given $\varepsilon>0$ there exists $R>0$ such that

$$
\|u\|_{L^{2}\left(\Omega \cap B_{R}^{c}\right)} \leq \varepsilon\|u\|_{\tilde{H}^{s, 2}(\Omega)}, \quad \text { for every } u \in \tilde{H}^{s, 2}(\Omega) .
$$

To prove Step 2 it is sufficient to consider $0<s<1$, thanks to Step 1 . 

get

We fix $\varphi \in C_{c}^{\infty}\left(B_{2}\right)$ such that $\varphi=1$ on $B_{1}$ and $0 \leq \varphi \leq 1$. Setting $\varphi_{r}(x)=\varphi\left(\frac{x}{r}\right)$ we

$$
\begin{aligned}
& \left\|\left(1-\varphi_{r}\right) u\right\|_{L^{2}\left(\mathbb{R}^{n}\right)}^{2}=\left\|\mathcal{F}\left(\left(1-\varphi_{r}\right) u\right)\right\|_{L^{2}\left(\mathbb{R}^{n}\right)}^{2} \\
& =\int_{|\xi|<R_{1}}\left|\mathcal{F}\left(\left(1-\varphi_{r}\right) u\right)\right|^{2} d \xi+\int_{|\xi| \geq R_{1}}\left|\mathcal{F}\left(\left(1-\varphi_{r}\right) u\right)\right|^{2} d \xi \\
& \leq \frac{1}{(2 \pi)^{n}}\left|B_{R_{1}}\right|\left(\int_{\mathbb{R}^{n}}\left|\left(1-\varphi_{r}\right) u\right| d x\right)^{2}+R_{1}^{-2 s} \int_{|\xi| \geq R_{1}}|\xi|^{2 s}\left|\mathcal{F}\left(\left(1-\varphi_{r}\right) u\right)\right|^{2} d \xi \\
& =: I_{1}+I_{2} .
\end{aligned}
$$

Using that $\operatorname{supp}\left(1-\varphi_{r}\right) u \subset \Omega \cap B_{r}^{c}$ and by Hölder inequality we bound

$$
I_{1} \leq \frac{1}{(2 \pi)^{n}}\left|B_{R_{1}}\right|\left|\Omega \cap B_{r}^{c}\right| \int_{\Omega \cap B_{r}^{c}}\left|\left(1-\varphi_{r}\right) u\right|^{2} d x \leq \frac{1}{(2 \pi)^{n}}\left|B_{R_{1}}\right|\left|\Omega \cap B_{r}^{c}\right|\|u\|_{L^{2}(\Omega)}^{2} .
$$

From [4, Proposition 3.4] we have

$$
\int_{\mathbb{R}^{n}}|\xi|^{2 s}|\hat{u}|^{2} d \xi=C(n, s) \int_{\mathbb{R}^{n}} \int_{\mathbb{R}^{n}} \frac{|u(x)-u(y)|^{2}}{|x-y|^{n+2 s}} d x d y
$$

and hence

$$
\begin{aligned}
I_{2} & \leq R_{1}^{-2 s} \int_{\mathbb{R}^{n}}|\xi|^{2 s}\left|\mathcal{F}\left(\left(1-\varphi_{r}\right) u\right)\right|^{2} d \xi \\
& =C_{0} R_{1}^{-2 s} \int_{\mathbb{R}^{n} \times \mathbb{R}^{n}} \frac{\left(\left(1-\varphi_{r}(x)\right) u(x)-\left(1-\varphi_{r}(y)\right) u(y)\right)^{2}}{|x-y|^{n+2 s}} d x d y \\
& =C_{0} R_{1}^{-2 s} \int_{\mathbb{R}^{n} \times \mathbb{R}^{n}} \frac{\left(\left(1-\varphi_{r}(x)\right)(u(x)-u(y))-u(y)\left(\varphi_{r}(x)-\varphi_{r}(y)\right)\right)^{2}}{|x-y|^{n+2 s}} d x d y \\
& \leq 2 C_{0} R_{1}^{-2 s} \int_{\mathbb{R}^{n} \times \mathbb{R}^{n}} \frac{\left(\frac{\left(1-\varphi_{r}(x)\right)^{2}(u(x)-u(y))^{2}}{|x-y|^{n+2 s}}+\frac{u^{2}(y)\left(\varphi_{r}(x)-\varphi_{r}(y)\right)^{2}}{|x-y|^{n+2 s}}\right) d x d y}{} \\
& \leq 2 C_{0} R_{1}^{-2 s} \int_{\mathbb{R}^{n} \times \mathbb{R}^{n}} \frac{(u(x)-u(y))^{2}}{|x-y|^{n+2 s}} d x d y+2 C_{0} R_{1}^{-2 s} \int_{\mathbb{R}^{n}} u^{2}(y) \int_{\mathbb{R}^{n}} \frac{\left(\varphi_{r}(x)-\varphi_{r}(y)\right)^{2}}{|x-y|^{n+2 s}} d x d y \\
& \leq C_{1} R_{1}^{-2 s}\left(\left\|(-\Delta)^{s} u\right\|_{L^{2}\left(\mathbb{R}^{n}\right)}^{2}+\|u\|_{L^{2}(\Omega)}^{2}\right),
\end{aligned}
$$

where in the last inequality we have used that

$$
\int_{\mathbb{R}^{n}} \frac{\left(\varphi_{r}(x)-\varphi_{r}(y)\right)^{2}}{|x-y|^{n+2 s}} d x \leq C, \quad y \in \mathbb{R}^{n}, r \geq 1 .
$$

Thus we have Step 2 by choosing $R$ so that $\left|B_{R_{1}}\right|\left|\Omega \cap B_{R}^{c}\right|<\frac{\varepsilon}{2}$ where $C_{1} R_{1}^{-2 s}=\frac{\varepsilon}{2}$.

Step 3 The embedding $\tilde{H}^{s, 2}(\Omega) \hookrightarrow L^{2}(\Omega)$ is compact for any $0<s<1$. 
Let us consider a bounded sequence $\left\{u_{k}\right\}_{k=1}^{\infty}$ in $\tilde{H}^{s, 2}(\Omega)$. Let $\varphi, \varphi_{\ell}$ be as in Step 2 (here $\ell \in \mathbb{N}$ ). Then for a fixed $\ell$ the sequence $\left\{\varphi_{\ell} u_{k}\right\}_{k=1}^{\infty}$ is bounded in $\tilde{H}^{s, 2}(\Omega)$ (the proof is very similar to the estimate of $I_{2}$ in Step 2).

Since the embedding $\tilde{H}^{s, 2}\left(B_{r}\right) \hookrightarrow L^{2}\left(B_{r}\right)$ is compact (see e.g. [4, Theorem 7.1]), there exists a subsequence $\left\{u_{k}^{1}\right\}_{k=1}^{\infty}$ such that $\varphi_{1} u_{k}^{1} \rightarrow u^{1}$ in $L^{2}\left(B_{2}\right)$. Inductively we will have $\varphi_{\ell} u_{k}^{\ell} \rightarrow u^{\ell}$ in $L^{2}\left(B_{2 \ell}\right)$ where $\left\{u_{k}^{\ell+1}\right\}_{k=1}^{\infty}$ is a subsequence of $\left\{u_{k}^{\ell}\right\}_{k=1}^{\infty}$ for $\ell \geq 1$. Moreover, we have $u^{\ell+1}=u^{\ell}$ on $B_{\ell}$. Setting $u=\lim _{\ell \rightarrow \infty} u^{\ell}$ it follows that $u_{k}^{k}$ converges to $u$ in $L^{2}(\Omega)$, thanks to Step 2 .

Step 4 The embedding $\tilde{H}^{s, 2}(\Omega) \hookrightarrow \tilde{H}^{r, 2}(\Omega)$ is compact for any $0 \leq r<s$.

Since the composition of two compact operators is compact, we can assume that $s-r<1$.

Let $\left\{u_{k}\right\}_{k=1}^{\infty}$ be a bounded sequence in $\tilde{H}^{s, 2}(\Omega)$. Setting $v_{k}=(-\Delta)^{\frac{r}{2}} u_{k}$ we see that $\left\{v_{k}\right\}_{k=1}^{\infty}$ is a a bounded sequence in $\tilde{H}^{s-r, 2}(\Omega)$. Then by Step 3 (up to a subsequence) $v_{k}$ converges to some $v$ in $L^{2}(\Omega)$ which is equivalent to saying that (up to a subsequence) $u_{k}$ converges to some $u$ in $\tilde{H}^{r, 2}(\Omega)$.

Finally, compactness of the embedding $\tilde{H}^{\frac{n}{2}, 2}(\Omega) \hookrightarrow L^{p}(\Omega)$ follows from the compactness of $\tilde{H}^{\frac{n}{2}}, 2(\Omega) \hookrightarrow L^{2}(\Omega)$, Theorem B and Lemma A.9.

Lemma A.8 (Exact constant) We set

$$
f(x)=\log \frac{1}{|x|}, \quad x \in \mathbb{R}^{n} .
$$

Then

$$
(-\Delta)^{\sigma} f(x)=\gamma_{n} 2^{2 \sigma-n} \pi^{-\frac{n}{2}} \frac{\Gamma(\sigma)}{\Gamma\left(\frac{n-2 \sigma}{2}\right)} \frac{1}{|x|^{2 \sigma}}, \quad 0<\sigma<\frac{n}{2},
$$

where $\Gamma$ is the gamma function and $\gamma_{n}=\frac{(n-1) !}{2}\left|S^{n}\right|$.

Proof. Using a rescaling argument one can get (see for e.g. [7, Lemma A.5])

$$
(-\Delta)^{\sigma} f(x)=(-\Delta)^{\sigma} f\left(e_{1}\right) \frac{1}{|x|^{2 \sigma}} \text {. }
$$

To compute the value of $(-\Delta)^{\sigma} f\left(e_{1}\right)$ we use the fact that $\frac{1}{\gamma_{n}} \log \frac{1}{|x|}$ is a fundamental solution of $(-\Delta)^{\frac{n}{2}}$ (see for instance [7, Lemma A.2]) i.e.,

$$
\int_{\mathbb{R}^{n}} \log \frac{1}{|x|}(-\Delta)^{\frac{n}{2}} \varphi(x) d x=\gamma_{n} \varphi(0), \quad \varphi \in \mathcal{S}\left(\mathbb{R}^{n}\right) .
$$


Using integration by parts, which can be verified, we obtain

$$
\begin{aligned}
\gamma_{n} \varphi(0) & =\int_{\mathbb{R}^{n}} f(x)(-\Delta)^{\frac{n}{2}} \varphi(x) d x \\
& =\int_{\mathbb{R}^{n}}(-\Delta)^{\sigma} f(x)(-\Delta)^{\frac{n}{2}-\sigma} \varphi(x) d x \\
& =\int_{\mathbb{R}^{n}} \frac{(-\Delta)^{\sigma} f\left(e_{1}\right)}{|x|^{2 \sigma}}\left(|\xi|^{n-2 \sigma} \widehat{\varphi}\right)^{\vee}(x) d x \\
& =(-\Delta)^{\sigma} f\left(e_{1}\right) \int_{\mathbb{R}^{n}}\left(\frac{1}{|x|^{2 \sigma}}\right)^{\vee}(\xi)\left(|\xi|^{n-2 \sigma} \widehat{\varphi}\right) d \xi \\
& =(-\Delta)^{\sigma} f\left(e_{1}\right) 2^{n-2 \sigma-\frac{n}{2}} \frac{\Gamma\left(\frac{n-2 \sigma}{2}\right)}{\Gamma\left(\frac{2 \sigma}{2}\right)} \int_{\mathbb{R}^{n}} \frac{1}{|\xi|^{n-2 \sigma}}\left(|\xi|^{n-2 \sigma} \widehat{\varphi}\right) d \xi \\
& =(-\Delta)^{\sigma} f\left(e_{1}\right) 2^{n-2 \sigma-\frac{n}{2}} \frac{\Gamma\left(\frac{n-2 \sigma}{2}\right)}{\Gamma\left(\frac{2 \sigma}{2}\right)}(2 \pi)^{\frac{n}{2}} \varphi(0),
\end{aligned}
$$

where in the 4th equality we have used that

$$
\mathcal{F}\left(\frac{1}{|x|^{n-\alpha}}\right)=2^{\alpha-\frac{n}{2}} \frac{\Gamma\left(\frac{\alpha}{2}\right)}{\Gamma\left(\frac{n-\alpha}{2}\right)} \frac{1}{|x|^{\alpha}}, \quad 0<\alpha<n,
$$

in the sense of tempered distribution. Since in our case $\mathcal{F}$ is the normalized Fourier transform, the constant in the right hand side of (22) appears slightly different from [12, Section 5.9].

Hence we have the lemma.

The following lemma is the Vitali's convergence theorem.

Lemma A.9 (Vitali's convergence theorem) Let $\Omega$ be a measure space with finite measure $\mu$ i.e., $\mu(\Omega)<\infty$. Let $f_{k}$ be a sequence of measurable function on $\Omega$ be such that

i) $f_{k} \stackrel{k \rightarrow \infty}{\longrightarrow} f$ almost everywhere in $\Omega$.

ii) For $\varepsilon>0$ there exists $\delta>0$ such that

$$
\int_{\tilde{\Omega}}\left|f_{k}\right| d \mu<\varepsilon \quad \text { for every } \tilde{\Omega} \subset \Omega \text { with } \mu(\tilde{\Omega})<\delta .
$$

Or,

$\left.i i^{\prime}\right)$ There exists $p>1$ such that

$$
\sup _{k \in \mathbb{N}} \int_{\Omega}\left|f_{k}\right|^{p} d \mu<\infty
$$

Then $f_{k} \rightarrow f$ in $L^{1}(\Omega)$.

Acknowledgements I would like to thank my advisor Prof. Luca Martinazzi for all the useful discussion and encouragement. 


\section{References}

[1] D. Adams: A sharp inequality of J. Moser for higher order derivatives, Ann. of Math. 128 (1988), 385-398.

[2] Adimurthi: Existence of positive solutions of the semilinear Dirichlet problem with critical growth for the n-Laplacian, Ann. Scuola Norm. Sup. Pisa Cl. Sci. 417 (1990), no. 3, 393413.

[3] Adimurthi, M. Struwe: Global compactness properties of semilinear elliptic equations with critical growth, J. Funct. Anal. 175 (2000), 125-167.

[4] E. Di Nezza, G. Palatucci, E. Valdinoci: Hitchhiker's guide to the fractional Sobolev spaces, Bull. Sci. Math. 136 (2012), No. 5, 521-573.

[5] O. Druet: Multibumps analysis in dimension 2: quantification of blow-up levels, Duke Math. J. 132 (2006), 217-269.

[6] L. Fontana, C. Morpurgo: Sharp Adams and Moser-Trudinger inequalities on $\mathbb{R}^{n}$ and other spaces of infinite measure, arXiv: 1504.04678 (2015).

[7] A. Hyder: Structure of conformal metrics on $\mathbb{R}^{n}$ with constant Q-curvature, arXiv: 1504.07095 (2015).

[8] A. Iannizzotto, M. Squassina: 1/2-Laplacian problems with exponential nonlinearity, J. Math. Anal. Appl. 414 (2014), 372-385.

[9] S. Iula, A. Maalaoui, L. Martinazzi: A fractional Moser-Trudinger type inequality in one dimension and its critical points, arXvi: 1504.04862 (2015).

[10] O. LAKKIS: Existence of solutions for a class of semilinear polyharmonic equations critical exponential growth, Adv. Diff. Eq. 4 No. 6 (1999), 877-906.

[11] N. Lam, G. Lu: A new approach to sharp Moser-Trudinger and Adams type inequalities: a rearrangement-free argument, J. Differential Equation 255 (2013), 298-325.

[12] E.H. Lieb, M. Loss: Analysis, Second edition. Graduate Studies in Mathematics, 14. American Mathematical Society, Providence, RI, 2001. ISBN:0-8218-2783-9.

[13] P. L. Lions: The concentration-compactness principle in the calculus of variations. The limit case. I., Rev. Mat. Iberoamericana. 1 (1985), No. 1, 145-201.

[14] A. Maalaoui, L. Martinazzi, A. Schikorra: Blow-up behaviour of a fractional AdamsMoser-Trudinger type inequality in odd dimension, arXiv: 1504.00254 (2015).

[15] A. Malchiodi, L. Martinazzi: Critical points of the Moser-Trudinger functional on a disk, J. Eur. Math. Soc. (JEMS) 16 (2014), 893-908.

[16] L. Martinazzi: A threshold phenomenon for embeddings of $H_{0}^{m}$ into Orlicz spaces, Calc. Var. Partial Differential Equations 36 (2009), 493-506. 
[17] L. Martinazzi: Fractional Adams-Moser-Trudinger type inequalities, Nonlinear Analysis 127 (2015), 263-278.

[18] L. Martinazzi, M. Struwe: Quantization for an elliptic equation of order $2 m$ with critical exponential non-linearity, Math Z. 270 (2012), 453-487.

[19] N. Masmoudi, F. Sani: Trudinger-Moser Inequalities with the Exact Growth Condition in $\mathbb{R}^{n}$ and Applications, Comm. Partial Differential Equations, 40 (2015), No. 8, 1408-1440.

[20] J. Moser: A sharp form of an inequality by N. Trudinger, Indiana Univ. Math. J. 20 (1970/71) 1077-1092.

[21] S. I. Pohozaev: On eigenfunctions of the equation $\Delta u+\lambda f(u)=0$, Dokl. Akad. Nank. SSSR 165 (1965), 36-39.

[22] F. Robert, M. Struwe: Asymptotic profile for a fourth order PDE with critical exponential growth in dimension four, Adv. Nonlin. Stud. 4 (2004), 397-415.

[23] B. RuF: A sharp Trudinger-Moser type inequality for unbounded domains in $\mathbb{R}^{2}$, J. Funct. Anal. 219 (2) (2005), 340-367.

[24] L. Silvestre: Regularity of the obstacle problem for a fractional power of the Laplace operator, Comm. Pure Appl. Math. 60 (2007), no. 1, 67-112.

[25] E. M. Stein: Singular integrals and differentiable properties of functions, Princeton Math. Ser. 30 Princeton University Press, Princeton, NJ, 1970, xiv+290 pp.

[26] M. STRUwe: Quantization for a fourth order equation with critical exponential growth, Math. Z. 256 (2007), 397-424.

[27] N. S. Trudinger: On embedding into Orlicz spaces and some applications, J. Math. Mech. 17 (1967), 473-483.

[28] V. I. Yudovich: On certain estimates connected with integral operators and solutions of elliptic equations, Dokl. Akad. Nank. SSSR 138 (1961), 805-808. 